Research Paper

\title{
The pro-angiogenesis effect of miR33a-5p/Ets-1/DKK1 signaling in ox-LDL induced HUVECs
}

\author{
Mingxue Di1,2, Yu Zhang', Renya Zeng', Xiaolin Liu', Weijia Chen"1, Meng Zhang1, Cheng Zhang1, \\ Mengmeng $\mathrm{Li}^{1 凶}$ and Mei Zhang ${ }^{1 凶}$ \\ 1. The Key Laboratory of Cardiovascular Remodeling and Function Research, Chinese Ministry of Education, Chinese National Health Commission and \\ Chinese Academy of Medical Sciences, The State and Shandong Province Joint Key Laboratory of Translational Cardiovascular Medicine, Department of \\ Cardiology, Qilu Hospital of Shandong University, Jinan, China. \\ 2. Department of Gerontology, The First Affiliated Hospital of Shandong First Medical University \& Shandong Provincial Qianfoshan Hospital.
}

$\triangle$ Corresponding authors: Dr. Mei Zhang, Department of Cardiology, Qilu Hospital, Shandong University, No.107, Wen Hua Xi Road, Jinan, Shandong, 250012, China. Tel.: +86 531 82169139; Fax: +86 531 86169356; E-mail: zhangmei@email.sdu.edu.cn (additional email: daixh@vip.sina.com); Dr. Mengmeng Li, Department of Cardiology, Qilu Hospital, Shandong University, No.107, Wen Hua Xi Road, Jinan, Shandong, 250012, China. Tel.: +86 53182169139 ; E-mail: zpyzlmm90128@163.com.

(C) The author(s). This is an open access article distributed under the terms of the Creative Commons Attribution License (https://creativecommons.org/licenses/by/4.0/). See http://ivyspring.com/terms for full terms and conditions.

Received: 2021.03.10; Accepted: 2021.09.21; Published: 2021.10.03

\begin{abstract}
Objective: Angiogenesis is involved in multiple biological processes, including atherosclerosis (AS) and cancer. Dickkopfl (DKK1) plays many roles in both tumors and AS and has emerged as a potential biomarker of cancer progression and prognosis. Targeting DKK1 is a good choice for oncological treatments. Many anticancer therapies are associated with specific cardiovascular toxicity. However, the effects of DKK1 neutralizing therapy on AS are unclear. We focused on how DKK1 affected angiogenesis in AS and ox-LDL-induced human umbilical vein endothelial cells (HUVECs).

Methods: ApoE-/- mice were fed a high-fat diet and then injected with DKK1i or DKK1 lentivirus to study the effects of DKK1. In vitro, promoter assays, protein analysis, database mining, dual-luciferase reporter assay (DLR), electrophoretic mobility shift assay (EMSA), chromatin immunoprecipitation (ChIP), and coimmunoprecipitation (co-IP) were used to study the mechanism of DKKI biogenesis. Cell migration and angiogenesis assays were performed to investigate the function and regulatory mechanisms of DKK1.

Results: DKK1 participated in angiogenesis both in the plaques of ApoE-/- mice by knockdown or overexpression of DKK1 and ox-LDL-induced HUVECs. DKK1 induced angiogenesis (increasing migration and capillary formation, inducing expression of VEGFR-2/VEGF-A/MMP) via the CKAP4/PI3K pathway, independent of $W n t / \beta$-catenin. ox-LDL increased the expression and nuclear transfer of Ets- 1 and c-jun, and induced the transcriptional activity of DKK1 in HUVECs. Ets-1, along with c-jun and CBP, could bind to the promoter of DKK1 and enhance DKK1 transcription. MiR33a-5p was downregulated in ox-LDL induced HUVECs and aortic artery of high-fat diet ApoE-/- mice. Ets-1 was a direct target of miR33a-5p. MiR33a-5p/Ets-1/ DKK1 axis contributed to angiogenesis.

Conclusions: MiR33a-5p/Ets-1/DKK1 signaling participated in ox-LDL-induced angiogenesis of HUVECs via the CKAP4/PI3K pathway. These new findings provide a rationale and notable method for tumor therapy and cardiovascular protection.
\end{abstract}

Key words: DKK1; Ets-1; miR33a-5p; angiogenesis; HUVECs

\section{Introduction}

Dickkopf-1 (DKK1), a secreted inhibitor of the canonical Wnt/ $\beta$-catenin pathway, plays complex cellular and biological roles in different diseases. DKK1 is overexpressed in bone pathologies and many cancers, has now emerged as a potential biomarker of cancer progression and prognosis for several types of malignancies [1], and has been shown to have immunosuppressive effects [2]. DKK1 has been widely investigated in oncology and is now considered a promising target for anticancer therapy 
[1]. For example, DKN-01 is an IgG4 clinical stage antibody that potently and specifically neutralizes human and murine DKK1 and was used in a recently completed promising study in combination with pembrolizumab in patients with gastric/ gastroesophageal junction cancer [3]. The treatment outcomes for a wide range of malignancies have improved remarkably due to the development of many novel anticancer therapies, including vascular endothelial growth factor inhibitors (VEGFIs). However, as a side effect, oncological treatment may increase the morbidity and mortality of cardiovascular diseases (CVDs), including via acceleration of atherosclerosis (AS) [4]. We confirmed that DKK1 induced endothelial cell (EC) dysfunction and AS [5]. Other data also suggest that DKK1 is an important driver of the initiation and progression of AS and a promising target for atheroprotection [6]. DKN-01 was evaluated in a phase I multicenter study for advanced tumor therapy, and better outcomes were associated with biomarkers of angiogenesis inhibition, which indicated the potential antiangiogenic and immunomodulatory activity of DKN-01 [7]. Because there exist cross-susceptibility factors and common targets between tumors and CVDs, elucidating the regulatory effect and molecular mechanism of DKK1 in EC angiogenesis will provide a theoretical basis and clinical reference for the identification of new effective intervention targets for antitumor drugs.

ECs, located on the surface of the vascular wall, are always vulnerable to various risk factors, such as hypertension and hyperlipidemia. In our previous study, we found that DKK1 induces endothelial dysfunction in plaques and human umbilical vein endothelial cells (HUVECs) [8]. Angiogenesis, which provides essential oxygen and nutrients for proliferation and metastasis, is an indispensable process for tumor growth and metastatic dissemination. Tumor angiogenesis has become a new and promising target for antitumor therapy. While angiogenesis also plays an important role in AS, we focused on the effect of DKK1 on angiogenesis.

DKK1 has two cysteine rich domains (CRDs): CRD-N and CRD-C. DKK1 binds to LRP6 and antagonizes the downstream canonical Wnt pathway. In addition, the CRD-N of DKK1 binds to cytoskeleton-associated protein 4 (CKAP4), and then, the intracellular segment recruits PI3K and activates AKT. DKK1 induces angiogenesis through Wnt/ $\beta$ catenin-dependent or Wnt/ $\beta$-catenin-independent mechanisms in tumor cells. However, the downstream mechanisms by which DKK1 induces angiogenesis in ox-LDL-induced HUVECs are unknown.

Studies have found several mechanisms upstream of DKK1, including histone modification, transcriptional changes, posttranscriptional changes and posttranslational modifications (phosphorylation, glycosylation) [9-12]. However, the upstream mechanism underlying the upregulation of DKK1 in ox-LDL-induced HUVECs is still unknown. Promoter analysis by genome bioinformatics methods (such as JASPAR and ALGGEN) revealed that the DKK1 promoter can bind via multiple possible sites to the transcription factors Ets-1, c-jun, c-fos and so on. Under ox-LDL stimulation, we found that Ets-1 was obviously upregulated compared with other predicted transcription factors. Moreover, Ets-1 siRNA severely inhibited the upregulation of ox-LDL induced DKK1. Ets-1, a member of the E26 transformation-specific (Ets) family, possesses a conserved domain (EBS) that recognizes GGAA/T [13]. Previous studies found that Ets-1 performed multiple functions in ECs: (1) Ets-1 directly regulated several vascular genes, such as Flt1, Tek, Kdr, Angpt2, Nrp1, vWF, PECAM1 and Cdh5, to promote angiogenesis [14]; (2) Ets-1 directly upregulated MMPs (MMP1, MMP3, MMP9 and MMP13) and $\beta 3$ integrin to promote migration in the nucleus $[15,16]$. However, it is unclear whether Ets-1 affects the function of ECs by regulating DKK1.

MicroRNAs are a class of noncoding singlestranded small RNA molecules that can specifically pair with the 3' untranslated region (3'-UTR) of target gene mRNAs to inhibit the expression of target genes through translational repression or mRNA degradation [17]. One miRNA can target one or more genes, and the regulatory mechanism of a miRNA may be different in different cells. MiRNAs are regulators of vascular endothelial functions and AS. With the aid of well-known programs (such as miRBase, STARBASE and TargetScan), we found that miR33a-5p was markedly downregulated under ox-LDL stimulation in HUVECs. Both DKK1 and Ets-1 are target genes of miR33a-5p. However, whether miR33a-5p can bind to the $3^{\prime}$-UTR of DKK1 and Ets-1 mRNA to regulate their translation is unknown. Studies have found that miR33a-5p may be related to macrophage lipid metabolism [18] and inhibition of tumor cell proliferation [19]. A previous study also found that DKK1 is regulated by miR33a in diabetic cardiomyopathy [20]. However, the mechanisms of action of miR33a-5p in ECs and the function of miR33a-5p in regulating Ets-1 and DKK1 are not yet clear.

Based on these findings, we hypothesized that miR33a-5p/Ets-1 participates in the regulation of ox-LDL-induced DKK1 expression in HUVECs. To test this idea, we investigated the underlying upstream mechanisms of DKK1 expression in 
HUVECs angiogenesis. Identifying these pathways will improve our understanding of the regulation of tumor angiogenesis and provide new methods to address cardiovascular toxicity in antitumor therapy. An in-depth study of the effect of DKK1 on angiogenesis will provide a solid theoretical basis for improving the development of drugs to treat tumors and reduce or even protect against AS. It is hoped that the relevant new drugs will play a better synergistic role in the treatment of tumors and AS in clinical application.

\section{Materials and Methods}

Please see the Major Resources Table in the Supplemental Materials.

\section{Ethics statement}

All in vivo protocols involving animal care and experiments complied with the Guide for Care and Use of Laboratory Animals published by the United States National Institutes of Health (NIH Publication, 8th Edition, 2011) and the Animal Management Rules of the Chinese Ministry of Health (Document No. 55, 2001). All in vivo experiments were approved by the Animal Care Committee of Shandong University. All in vitro experimental protocols were approved by the Key Laboratory of Cardiovascular Remodeling and Function Research, Qilu Hosipital, China.

\section{Atherosclerosis animal model protocol and lentiviral gene transfer}

A total of 120 ApoE-/- mice (eight- week- old males) were purchased from Beijing HFK Bioscience Co.,Ltd. All mice were fed atherogenic chow (i.e., a high-fat diet with $0.25 \%$ cholesterol and $15 \%$ cocoa butter) at 14 weeks. The atherosclerotic model was created as previously described. We applied constrictive silica collars to the right carotid artery (RCA) to accelerate atherosclerotic lesion formation at the third week. Pentobarbital sodium was used for anesthesia via intraperitoneal injection $(40 \mathrm{mg} / \mathrm{kg})$ when placing the constrictive collars. Eight weeks after the surgery, the mice were randomly divided into four groups ( $\mathrm{n}=15$ each): a normal saline group (NS), an empty lentivirus group (GFP), a DKK1i lentivirus group (shDKK1), and a DKK1 lentivirus group (DKK1). A $200 \mu \mathrm{l}$ suspension $\left(4 \times 10^{8}\right.$ TU DKK1i or DKK1 lentivirus per $\mathrm{ml}$ ) was injected into each mouse through the tail vein. The mice were sacrificed 4 weeks posttransfection using pentobarbital sodium (50 mg/ kg, i.p.) before exsanguination by perfusion via the abdominal aorta with PBS.

\section{Histopathology and immunohistochemistry}

The RCAs were dissected, removed, fixed in $4 \%$ formaldehyde overnight at $4{ }^{\circ} \mathrm{C}$, and embedded in OCT compound, and 5 - $\mu$ m-thick sections were prepared. After blocking in 5\% bovine serum albumin (BSA) in PBS, the cryosections were incubated with primary antibodies overnight at $4^{\circ} \mathrm{C}$ and then with an HRP Detection System (ZSGB-BIO). Detection was subsequently performed using 3, 3'-diaminobenzidine (DAB) (ZSGB-BIO). Plaques stained with picrosirius red were viewed under polarized light. Staining in the plaque was quantified using Image-Pro Plus 6.0 software (Media Cybernetics, USA) and a color CCD video microscope (Olympus, Japan).

\section{Cell culture}

HUVECs were obtained from ScienCell Research Laboratories (Carlsbad, CA, USA) and cultivated in endothelial cell medium (ECM) (ScienCell, Carlsbad, CA). HEK293T cells were obtained from the American Type Culture Collection (ATCC) and cultured in high-glucose Dulbecco's modified Eagle's medium (DMEM). The medium contained $10 \%$ fetal bovine serum (FBS) and 1\% penicillin/streptomycin. Both cell lines were incubated in a humidified $5 \% \mathrm{CO}_{2}$ incubator at $37^{\circ} \mathrm{C}$.

\section{Plasmid construction}

Constructs with the wild-type and mutated 3'-UTRs of the human DKK1 gene (pGL3-DKK1-3'UTR-WT and pGL3-DKK1-3'-UTR-MUT) and the wild type and mutated 3'-UTRs of the human Ets-1 gene (pGL3-Ets-1-3'-UTR-WT and pGL3-Ets-1-3'UTR-MUT) were generated. The luciferase reporter gene plasmid (pGL3) and plasmids with the PGL3basic-DKK1 promoter (P0), the PGL3-DKK1 promoter (P1-P9), and the PGL3-basic-DKK1 promoter (delet ATGGAAT) (P0-del) were constructed, including the full-length DKK1 promoter, the serially truncated DKK1 promoter and deletion fragments of the DKK1 promoter, respectively. The PCDNA3.1-Ets-1 and PCDNA3.1-c-jun plasmids were constructed to overexpress Ets- 1 and c-jun, respectively. All the plasmids were obtained from Shanghai GenePharma Biotechnology Company.

\section{siRNA and RNA interference}

Upon reaching 40\%-60\% confluence, cells were transfected with specific siRNA or plasmids (GenePharma, Shanghai) using Lipofectamine 3000 (Thermo Fisher Scientific, USA) in Opti-MEM (Gibco, Thermo Fisher Scientific, USA). At $6 \mathrm{~h}$ after transfection, the medium was replaced. Cells were collected for detection of the luciferase reporter or of protein expression for $24-48 \mathrm{~h}$ after transfection.

\section{Immunofluorescence staining and microscopy}

The cells were washed with PBS, fixed with $4 \%$ 
paraformaldehyde, penetrated with $0.5 \%$ Triton X-100 PBS, and incubated with primary antibodies at $4{ }^{\circ} \mathrm{C}$ overnight. The sections were washed with PBS, and incubated with FITC- conjugated secondary antibodies. Nuclei were stained with 4', 6-diamidino2-phenylindole (DAPI; 1:2000, Roche, Germany) for 5 min. The samples were rinsed three times in PBS and examined under an epifluorescence microscope, and the data were analyzed using Image-Pro Plus 6.0 software (Media Cybernetics, USA).

\section{Western blot analysis}

HUVECs were lysed using RIPA buffer containing $1 \mathrm{mM}$ PMSF and collected by centrifugation at $14,000 \times \mathrm{rpm}$ for $10 \mathrm{~min}$. Proteins were separated on $10 \%$ SDS-PAGE gels, transferred to PVDF membranes with a $0.45 \mu \mathrm{m}$ pore size (Millipore, USA), and incubated with primary antibodies overnight at $4{ }^{\circ} \mathrm{C}$. The membranes were incubated with secondary antibodies the next day for $80 \mathrm{~min}$. Bands were visualized using Immobilon ECL substrate (Millipore, USA), and imaged with an LAS-4000 luminescent image analyzer (Fujifilm, USA). Protein expression was quantified using Adobe Photoshop CS6 (Adobe Systems, USA) and normalized to $\beta$-actin expression in each sample; the expression level is shown as a percentage of the control.

\section{RNA extraction and quantitative real-time PCR}

Total RNA was extracted from HUVECs using TRIzol reagent (Ambion, Life Technologies, USA), and reverse-transcribed into cDNA using a PrimeScript ${ }^{\mathrm{TM}}$ RT Reagent Kit (TakaRa Biotechnology, Dalian, China). cDNA (1 ng) was subjected to q-PCR using SYBR Green (TakaRa Biotechnology, Dalian, China) for the relative quantification of mRNA expression. Quantification was accomplished using the $2-\Delta \Delta \mathrm{Ct}$ method. $\beta$-Actin was used to normalize mRNA levels. U6 was used to normalize microRNA levels.

\section{Dual-luciferase reporter assay}

Cells were seeded in 24-well plates and cotransfected with $100 \mathrm{ng}$ of reporter plasmid and 20 ng of pRL-TK by using Lipofectamine 3000 (Thermo Fisher Scientific, USA) in Opti-MEM (Gibco, Thermo Fisher Scientific, USA). After $48 \mathrm{~h}$, cells (HUVECs or 293T cells) were harvested for the dual-luciferase assay. A dual-luciferase reporter (DLR) assay system (Genecopeia, USA) was used to measure the luciferase activity. The firefly luciferase values were normalized to Renilla luciferase activity before statistical analyses.

\section{Electrophoretic mobility shift assay}

HUVECs were treated with ox-LDL for $6 \mathrm{~h}$. Nuclear extracts were obtained using a NE-PER nuclear protein extraction kit (Thermo Scientific, Rockford IL, USA) according to the manufacturer's instructions. Double-stranded oligonucleotides were obtained by annealing equal amounts $(0.1 \mathrm{mg})$ of the complementary single-stranded oligonucleotides by heating to $95^{\circ} \mathrm{C}$ for $5 \mathrm{~min}$ and then gradually cooling to room temperature. Then, $0.01 \mu \mathrm{mol}$ of digoxigenin-labeled oligonucleotide probes was incubated with nuclear extracts in DNA binding buffer [10 mM Tris- $\mathrm{HCl}$ ( $\mathrm{pH} 7.5), 1 \mathrm{mM} \mathrm{MgCl}, 50 \mathrm{mM}$ $\mathrm{NaCl}, 0.5 \mathrm{mM}$ EDTA, $4 \%$ glycerol, and $0.5 \mathrm{mM}$ 2,3-dihydroxy-1,4-dithiobutane (DTT)] and $1 \mu \mathrm{g}$ of poly(dI-dC). A competition assay was performed using a 200-fold excess of cold probes or cold mutated probes $(2 \mu \mathrm{mol})$, which were preincubated with the reaction mixture before the addition of biotin-labeled probes. To ascertain the specificity of the nuclear proteins bound to Ets-1 sites, a supershift assay was performed with $2 \mathrm{mg}$ of Ets-1 antibody. After incubation for $30 \mathrm{~min}$, DNA-protein complexes were separated by $6.0 \%$ nondenaturing PAGE (Invitrogen) and transferred to a nylon membrane. DNA was crosslinked by UV irradiation for $10 \mathrm{~min}$. The nitrocellulose membrane was evaluated by the addition of a streptavidin-horseradish peroxidase conjugate and a chemiluminescent substrate. Then, the nitrocellulose membrane was imaged with an LAS-4000 luminescent image analyzer (Fujifilm, USA). A prominent single supershifted band was observed when nuclear extracts were incubated with an anti-Ets-1 antibody.

\section{Chromatin immunoprecipitation}

Chromatin immunoprecipitation (ChIP) analysis was performed by a ChIP kit (CST, Boston, USA), according to the manufacturer's protocols. Cells $\left(4 \times 10^{7}\right)$ were crosslinked with $4 \%$ formaldehyde, lysed, and enzymatically digested into 200-bp DNA fragments. The sheared chromatin was incubated with different antibodies and magnetic beads at $4^{\circ} \mathrm{C}$ overnight. Purified immunoprecipitated chromatin fragments from the IP samples were tested by PCR. The primers for the Ets-1 binding site in the human DKK1 promoter (-2080 to -1894) were as follows: 5 'ACACAGCTTGCAGATTTCCTAGT $-3{ }^{\prime}$ and 5 '-TATGGTCTGTGTTCTAGTTCCTTCA -3'. qPCR was used for quantitative analysis of the ChIP enrichment efficiency and for expression analysis according to the $2^{-\triangle \Delta C T}$ method. 


\section{Coimmunoprecipitation assay}

Cells $\left(4 \times 10^{7}\right)$ were lysed with $1 \mathrm{ml}$ of RIPA for 30 min and centrifuged at $12000 \times \mathrm{g}$ and $4^{\circ} \mathrm{C}$ for $30 \mathrm{~min}$. Save $10 \mu$ supernatant and added to $2 \times$ SDS loading buffer, denatured at $99{ }^{\circ} \mathrm{C}$ for $10 \mathrm{~min}$ standby. Pretreated protein A/G magnetic beads (Bimake, Shanghai, China) with $300 \mu \mathrm{l}$ RIPA were added to $6 \mu \mathrm{g}$ of antibody and then incubated $4{ }^{\circ} \mathrm{C}$ for 15 minutes. The mixture was placed on a magnet for 1 minute, removed the supernatant, and washed 3 times. The beads were resuspended by the sample $(300 \mu \mathrm{l})$, then incubated at $4{ }^{\circ} \mathrm{C}$ overnight with gentle rotation. The mixture was placed on a magnet for 1 minute, removed the supernatant, and washed 3 times. The beads was added to $1 \times$ SDS loading buffer, denatured at $99{ }^{\circ} \mathrm{C}$ for $10 \mathrm{~min}$, centrifuged $12000 \times \mathrm{g}$ for $10 \mathrm{~min}$, saved supernatant and evaluated by Western blot.

\section{EdU cell proliferation assay}

Five-ethynyl-2'-deoxyuridine (EdU) cell proliferation assays were carried out according to the manufacturer's instructions (RiboBio, Guangzhou, China) using the Cell-Light ${ }^{\mathrm{TM}}$ EdU imaging detection kit.

\section{Transwell assay}

Transwell inserts are an array of 24 individual Boyden chambers with $8 \mu \mathrm{m}$ pore size Transwell membranes (Corning, NY, USA). Cells were digested with trypsin and suspended in serum-free culture medium at $5 \times 10^{5} /$ well. Samples $(200 \mu \mathrm{l})$ were placed in the upper chamber, and the lower chamber was filled with serum-free culture medium $(500 \mu \mathrm{l})$. The cells were transfected and treated with ox-LDL at the designated concentrations and for the indicated times. The noninvading cells remained on the upper surface of the membrane and were removed with cotton swabs, whereas the cells that passed through the membrane were fixed with $4 \%$ formaldehyde, stained with $0.2 \%$ crystal violet, and then counted under an optical microscope after $24 \mathrm{~h}$.

\section{Scratch wound assay}

A thin mark was drawn vertically with a $20-\mu 1$ pipette tip in the six-well plate. The cells were then washed three times with PBS to remove the floating and detached cells. Fresh serum-free medium was added. The migratory distance was measured $0 \mathrm{~h}, 6 \mathrm{~h}$, $12 \mathrm{~h}$, and $24 \mathrm{~h}$ after wounding using IPP software. Cell migration is expressed as the percentage of the open wound area at $24 \mathrm{~h}$ relative to that at $0 \mathrm{~h}$.

\section{In vitro angiogenesis assay}

Cells were transfected or treated with ox-LDL. A 12-well plate was precoated with Matrigel (BD Bioscience, Billerica, MA, USA). Cells were digested with trypsin, suspended in serum-free culture medium at $5 \times 10^{4}$ /well and seeded on the 12-well plates. The cells were stained by calcein-AM, and the angiogenic properties were assessed after $12 \mathrm{~h}$. The tube length was measured using IPP software.

\section{Statistical analysis}

The data were analyzed using SPSS v23.0 (SPSS Inc., Chicago, IL) and are presented as the mean \pm SEM. of at least three independent experiments. Comparisons were analyzed using Student's $t$ test or one-way ANOVA followed by the Bonferroni post hoc test. $\mathrm{p}<0.05$ was considered statistically significant.

\section{Results}

\section{DKK1 aggravated plaque-associated angiogenesis in vivo}

The results of immunohistochemical analysis of DKK1 demonstrated that DKK1 protein expression was significantly lower in the shDKK1 group and higher in the DKK1 group than in the NS and GFP groups (Figure 1A), which established that the overexpression and silencing vectors were effective. In the immunohistochemical analysis, the intraplaque expression of VEGF-A, VEGFR-2, MMP-2 and MMP-9 was intense in the control group but was significantly downregulated with DKK1 knockdown, and upregulated with DKK1 overexpression (Figure 1B). Immunohistochemical analysis of the intraplaque expression of CD31 further confirmed that DKK1 increased plaque-associated angiogenesis (Figure 1C).

\section{DKK1 participated in angiogenesis in ox-LDL- induced HUVECs via the CKAP4/PI3K pathway}

HUVECs were treated with ox-LDL for different durations $(0 \mathrm{~h}, 1 \mathrm{~h}, 3 \mathrm{~h}, 6 \mathrm{~h})$. Western blotting and PCR showed that HUVECs treated with ox-LDL had increased DKK1 expression (Figure 2A and 2B). Compared with the NC+ox-LDL group, the si-DKK1+ox-LDL group showed less migration and tube formation (Figure 2C-2E). siRNA transfection downregulated the expression of DKK1 and inhibited the ox-LDL-induced upregulation of VEGF-A, VEGFR-2, MMP-2 and MMP-9 (Figure 2F). There was no difference between the NC+ox-LDL group and the si-DKK1+ox-LDL group in the EdU examination (Figure S1A). The results indicated that DKK1 is involved in migration and angiogenesis but not proliferation in ox-LDL-treated HUVECs. 

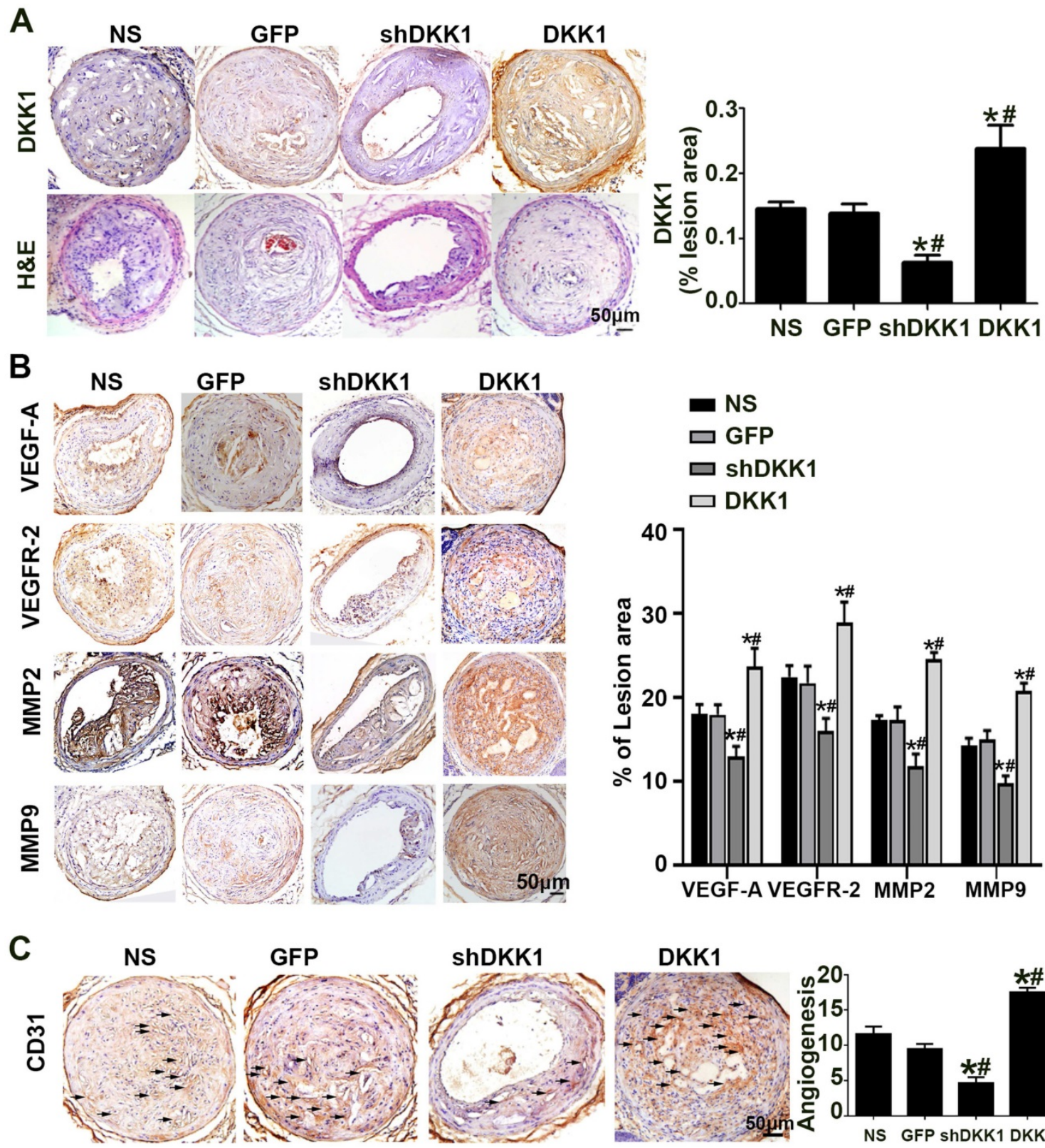

Figure 1. Effect of DKK1 on angiogenesis in the plaques of ApoE-/- mice. (A) Morphology of the RCAs, as shown by HE staining, and expression of DKK1 shown by DKK1. Bars indicate $50 \mu \mathrm{m}$. (B) Protein expression levels of VEGF-A, VEGFR-2, MMP-2 and MMP-9 in atherosclerotic plaques of ApoE-/- mice were determined by immunohistochemical staining. Bars indicate $50 \mu \mathrm{m}$. (C) Representative Immunohistochemically stained images and quantification of CD31 to measure new vessels in carotid plaques. Bars indicate $50 \mu \mathrm{m}$. The data are shown as the mean \pm SEM, $n=6$. $* P<0.05$ vs. NS; \#P<0.05 vs. GFP. RCA, right carotid arteries. NS, normal saline mice; GFP, lentivirus-GFP mice; shDKK1, lentivirus DKK1 shRNA; DKK1, lentivirus DKK1.

IM-12 is an agonist of the canonical Wnt pathway. There was no difference in angiogenesis markers (VEGF-A, VEGFR-2, MMP-2 and MMP-9) between the DKK1 group and the DKK1+IM-12 group (Figure S1B). In the DKK1 group, the expression of CKAP4 was upregulated (Figure 3A). siRNA transfection downregulated the expression of CKAP4 and inhibited the ox-LDL-induced upregulation of angiogenesis markers (VEGF-A, VEGFR-2, MMP-2 and MMP-9), while 740 Y-P, a PI3K agonist, restored the upregulation (Figure 3A). Compared with the DKK1 group, the DKK1+si-CKAP4 group showed less migration and tube formation, while 740 Y-P restored the migration and tube formation (Figure 3B-3D). The results indicate that DKK1/CKAP4/PI3K is involved in angiogenesis in ox-LDL-treated HUVECs.

\section{Ets- 1 participated in the ox-LDL-induced upregulation of DKK1 in HUVECs at the transcriptional level}

HUVECs were treated with ox-LDL for different durations ( 0 h, 1 h, 3 h, 6 h). Western blot and PCR showed that HUVECs treated with ox-LDL had higher Ets-1 expression (Figure S2A and S2B) than the $0 \mathrm{~h}$ group. Immunofluorescence showed that the 
nuclear translocation of Ets-1 increased after ox-LDL treatment (Figure S2C). siRNA transfection downregulated the expression of Ets-1 and inhibited the ox-LDL-induced upregulation of DKK1 (Figure
4A and 4B). The results indicate that Ets-1 is involved in the regulation of DKK1 expression in ox-LDL-treated HUVECs.
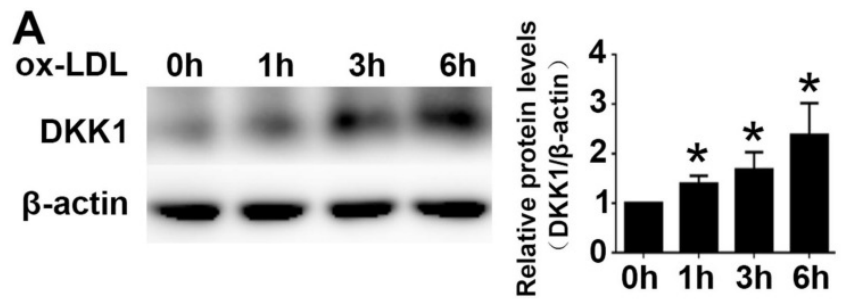
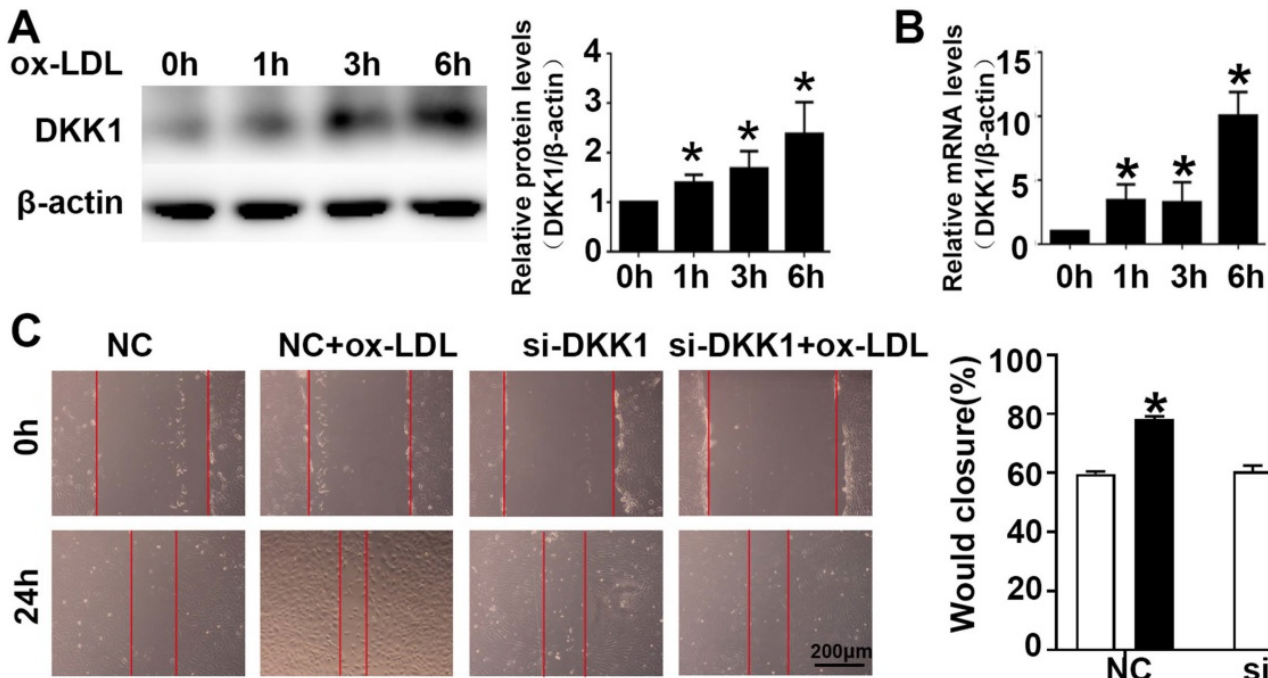

NC+ox-LDL
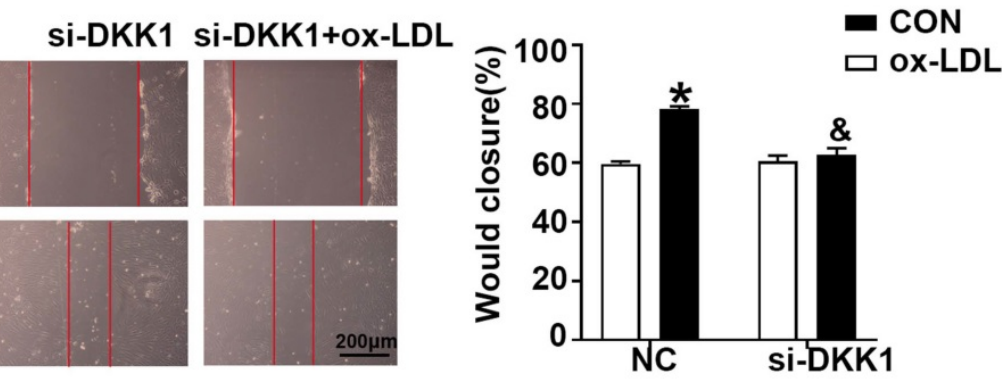

D

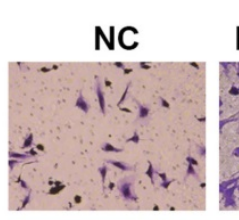

NC+ox-LDL
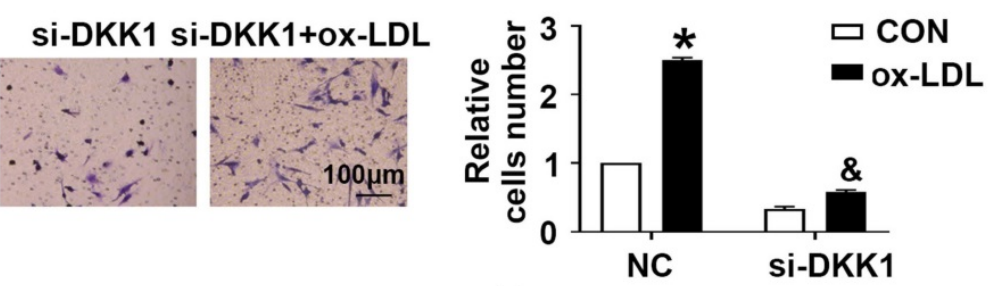

E

NC
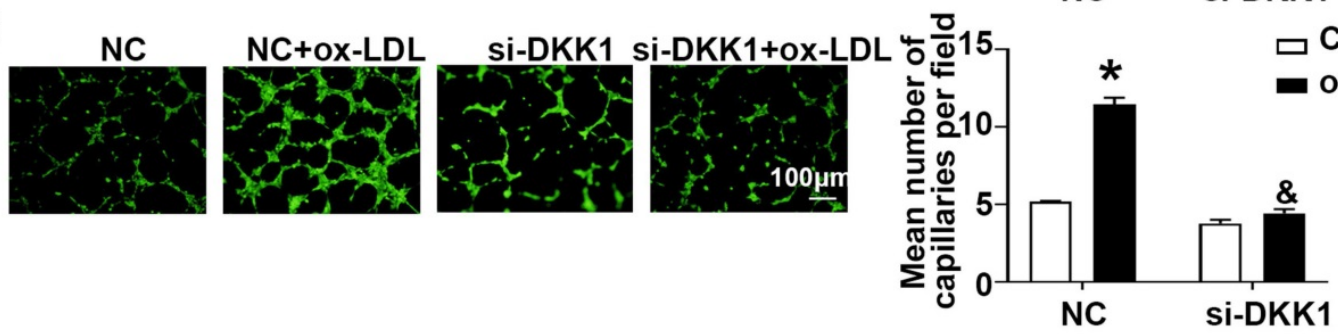

$\mathbf{F}$
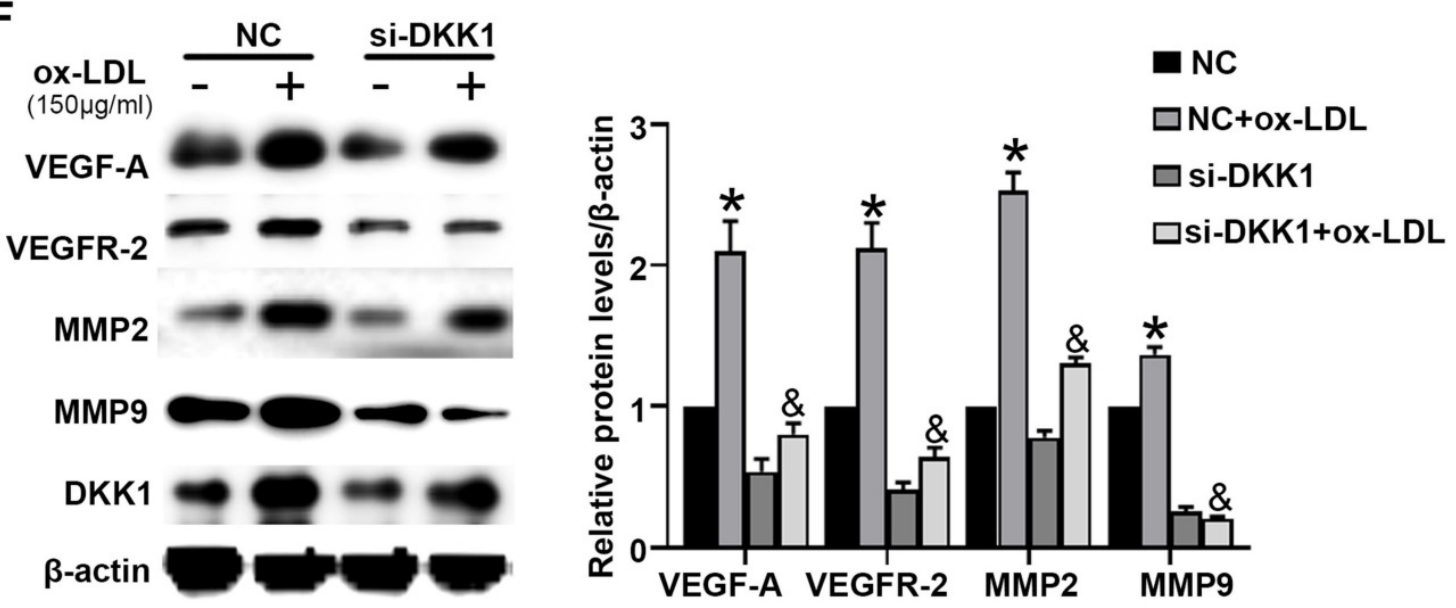

Figure 2. Time-dependent effects of ox-LDL treatment on the expression of DKK1 and DKK1 participates in antiangiogenic effects in ox-LDL induced HUVECs. (A-B) Quantification of the DKK1 level in HUVECs treated with ox-LDL $(150 \mu \mathrm{g} / \mathrm{ml})$ for various durations $(0 \mathrm{~h}, 1 \mathrm{~h}, 3 \mathrm{~h}, 6 \mathrm{~h})$ : (A) DKK1 protein expression levels. (B) DKK1 mRNA levels. (C-F) HUVECs were transiently transfected with the negative control (NC) and DKK1 siRNA (si-DKK1) for $24 \mathrm{~h}$ and then treated with $150 \mu \mathrm{g} / \mathrm{ml}$ ox-LDL for $6 \mathrm{~h}$. (C) Representative images and quantification of cell migration in the in vitro scratch wound assay (relative to $0 \mathrm{~h}$ ) at $0 \mathrm{~h}$ and $24 \mathrm{~h}$ post-wounding. Bars indicate 200 $\mu \mathrm{m}$. (D) Representative images and quantification of cell migration in Transwell assays. The cell counts on the bottom of the Transwell are shown here. Bars indicate $100 \mu \mathrm{m}$. (E) Representative image and quantification of tube length (\% of control). Bars indicate $100 \mu \mathrm{m}$. (F) Western blot to quantify VEGF-A, VEGF-2, MMP2 and MMP9 protein levels. The data are shown as the mean \pm SEM. $n=6$. $* P<0.05$ vs. the untreated group or NC; \&P<0.05 vs. NC+ ox-LDL. 
A
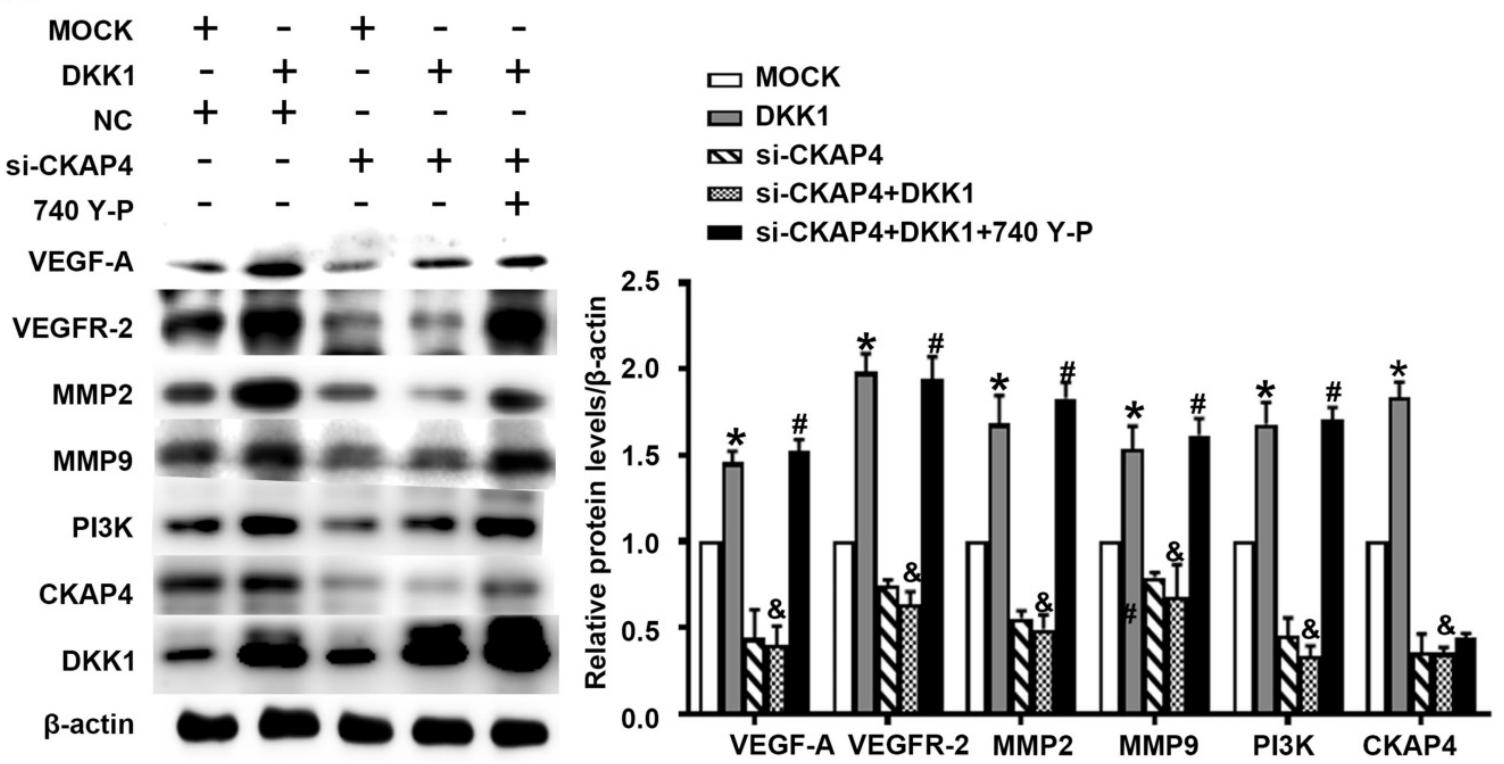

B
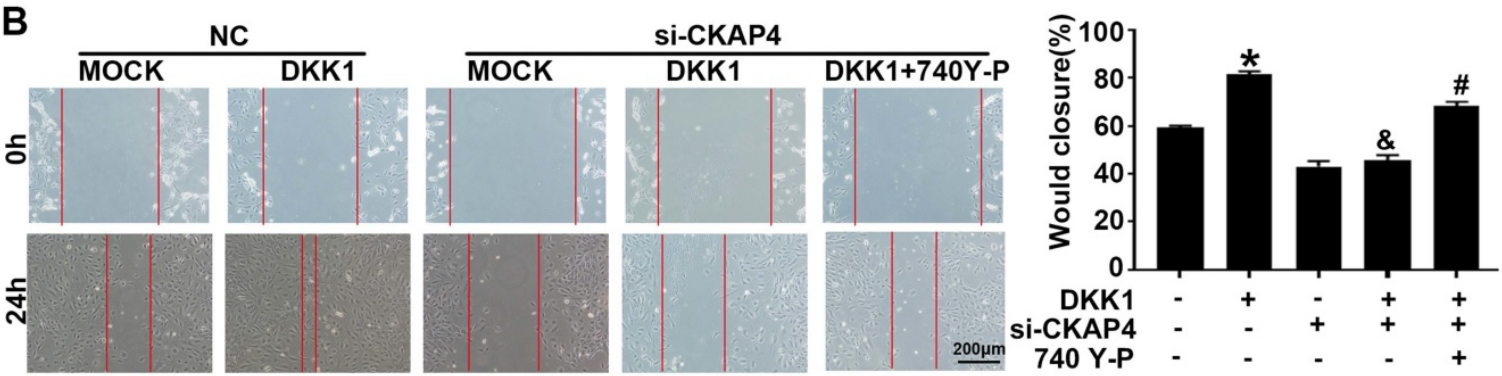

C
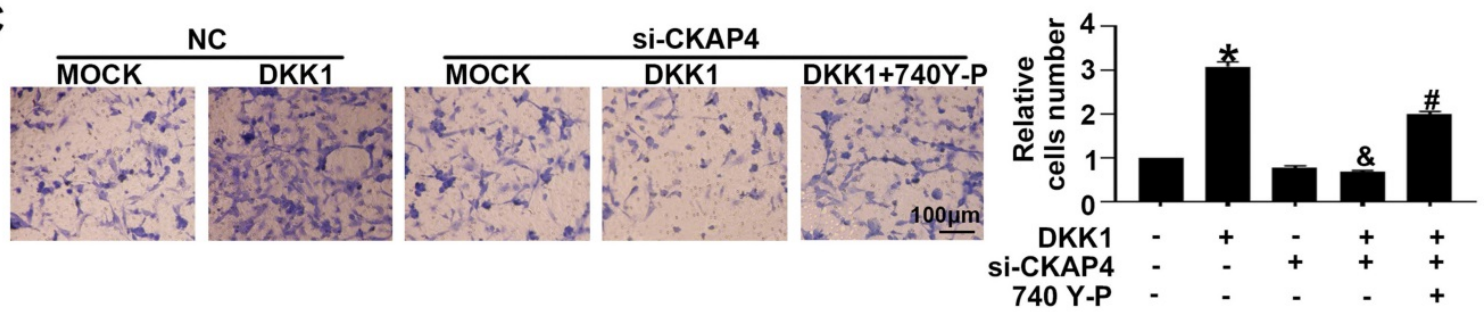

D
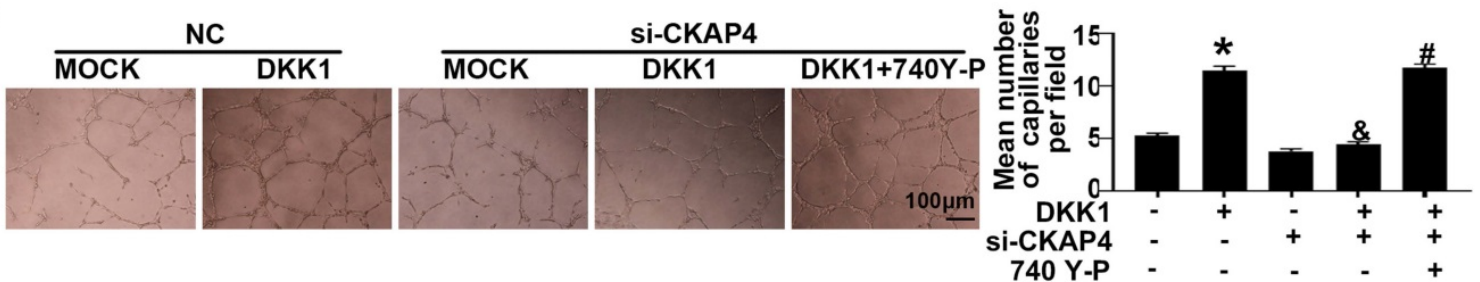

Figure 3. DKK 1 induces migration and angiogenesis by promoting CKAP4/PI3K. Cells were pretreated with PBS or $740 \mathrm{Y}-\mathrm{P}(30 \mu \mathrm{M})$ for $12 \mathrm{~h}$ before transfection with lenti-DKK1 and DKK1 siRNA (si-DKK1) for $24 \mathrm{~h}$. (A) Western blot for quantification of PI3K,VEGF-A.VEGF-2,MMP2 and MMP9 protein levels. (B) Representative images and quantification of cell migration in the in vitro scratch wound assay (relative to $0 \mathrm{~h}$ ) from $0 \mathrm{~h}$ and $24 \mathrm{~h}$ post-wounding. Bars indicate $200 \mu \mathrm{m}$. (C) Representative images and quantification of cell migration in Transwell assays. The cell counts on the bottom of the Transwell are shown here. Bars indicate $100 \mu \mathrm{m}$. (D) Representative image and quantification of the tube length (\% of control). Bars indicate $100 \mu \mathrm{m}$. The data are shown as the mean \pm SEM. $n=6$. $* P<0.05$ vs. MOCK; \&P<0.05 vs. DKK1 transfection only; $\# P<0.05$ vs. DKK1 and si-CKAP4 cotransfection.

Full-length, serially truncated and deletion fragment versions of the DKK1 promoter were cloned into the luciferase reporter vector pGL3-basic to generate $\mathrm{pGL3}$ - DKK1-promoter vectors, which were named P0, P1-P9 and P0-del, respectively. HUVECs were transiently cotransfected with the P0 and
pRL-TK vectors and then exposed to ox-LDL for $6 \mathrm{~h}$. A DLR assay showed that ox-LDL significantly increased the DKK1 promoter activity in HUVECs compared with the control. This finding indicated that ox-LDL could regulate DKK1 expression at the transcriptional level (Figure 4C). 
A

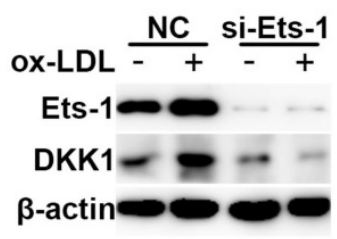

D

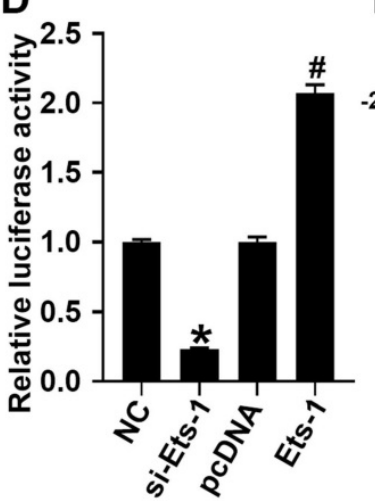

B

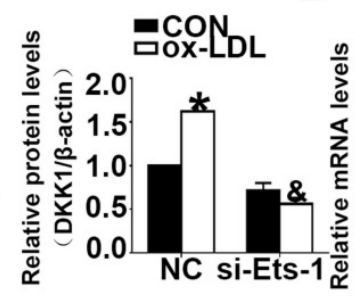

E

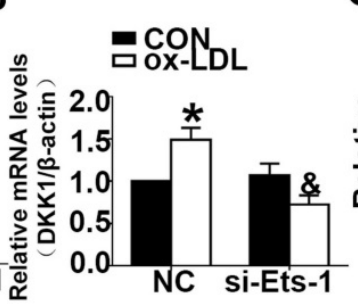

C

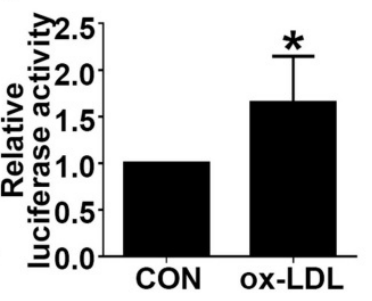

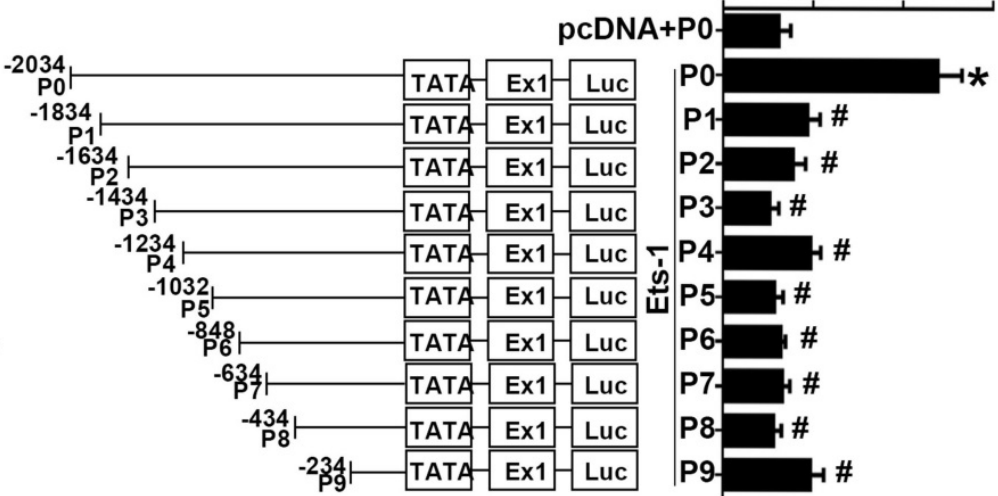

F

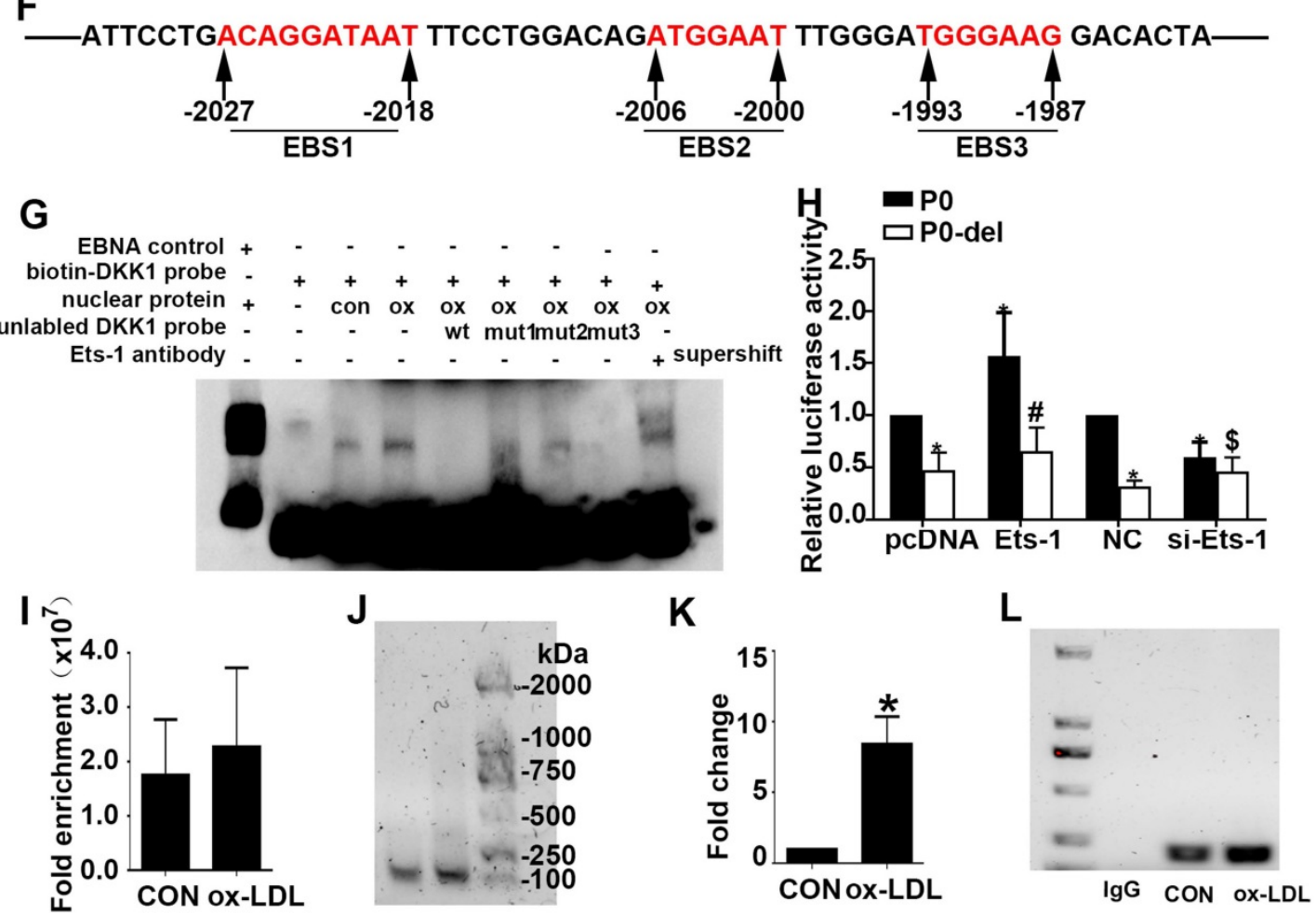

Figure 4. Ets-1 promotes the expression of DKK1 by binding with a key positive regulatory region of the human DKK1 promoter. (A-B) HUVECs were transiently transfected with the negative control (NC) and Ets-1 siRNA (si-Ets-1) for $24 \mathrm{~h}$ and then treated with $150 \mu \mathrm{g} / \mathrm{ml}$ ox-LDL for $6 \mathrm{~h}$ : (A) Western blot to quantify DKK1 protein levels; (B) qPCR to quantify DKK1 mRNA levels. (C) DLR assay in HUVECs transfected with the full-length DKK1 promoter and then treated with $150 \mu g / m l$ ox-LDL for $6 \mathrm{~h}$. (D) DLR assay in 293T cells transfected with si-Ets-1 or PCDNA3.1-Ets-1 and the full-length DKK1 promoter. (E) DLR assay in 293T cells transfected with PCDNA3.1-Ets-1 and the larger serial fragment from -2034 to $-234 \mathrm{bp}$ of the DKK1 promoter. (F) Prediction of Ets-1 binding sites in this region (-2034 -1834bp) of the DKK1 promoter: EBS1 (-2023 to -2018bp), EBS2 (-2006 to -2000bp) and EBS3 (-1993 to -1987bp). (G) The oligonucleotides from -2036 to $-1985 \mathrm{bp}$ of the DKK1 promoter were labeled with digoxin and incubated with nuclear extracts in the absence or presence of a 200 -fold excess of unlabeled wild-type oligonucleotides or mutant oligonucleotides (mut 1: mutant of the EBS1 element, mut2: mutant of the EBS2 element, and mut3: mutant of the EBS3 element). DNA-protein complexes were resolved by nondenaturing PAGE. (H) DLR assay in 293T cells transfected with si-Ets-1 (Ets-1 siRNA) or Ets-1 (PCDNA3.1-Ets-1 plasmids) and the deletion fragment promoter (-2006 -2000bp) for 48 h. (I-L) The results of ChIP analysis show that Ets-1 was recruited to the DKK1 promoter regions in HUVECs. IgG was used as an immunoprecipitation control. The immunoprecipitated DNA was evaluated by real-time PCR and agarose gel electrophoresis. The data are presented as the mean $\pm S E M$. $n=6$. $* P<0.05$ vs. the control group or NC; \&P<0.05 vs. NC+ ox-LDL; $\# P<0.05$ vs. P0+ Ets-1; $\$ P<0.05$ vs. P0+ si-Ets-1. PCDNA3.1 indicates basic plasmids, Ets-1 indicates PCDNA3.1-Ets-1 plasmids. NC indicates the negative control, si-Ets- 1 indicates Ets-1 siRNA. CON indicates the untreated HUVEC group, ox-LDL indicates HUVECs treated with ox-LDL. 
Cultured 293T cells were transiently cotransfected with the P0 and pRL-TK vectors and then transfected with PCDNA3.1-Ets-1, PCDNA3.1, negative control (NC) siRNA or Ets-1 siRNA. A DLR assay was performed and showed that Ets-1 siRNA decreased the DKK1 promoter activity in 293T cells (Figure 4D) and that Ets-1 significantly enhanced the promoter activity in 293T cells (Figure 4D).

To identify a plausible regulatory promoter region, we generated a series of $5^{\prime}$ deleted luciferase reporter constructs containing $\mathrm{P}(-2034,1834,1634$, $1434,1234,1032,848,634,434,234) /$ luc fragments of the DKK1 promoter (groups P0 -P9). Cultured 293T cells were transiently cotransfected with the $\mathrm{P} 0$ vector, pRL-TK vector and PCDNA3.1 (control group) or cotransfected with the P0-P9 vectors, $\mathrm{pRL}-\mathrm{TK}$ vector and PCDNA3.1-Ets1 (groups P0-P9). Compared to the control group, the P0 group showed a more than a 4-fold change, while the activity of the DKK1 promoter was significantly reduced from the P1 group to the P9 group (Figure 4E).

To identify putative cis-acting elements and transcription factors contributing to DKK1 promoter activities in the region spanning -2034 to $-1834 \mathrm{bp}$, the online prediction tools JASPAR and PROMO were used. Three possible loci, namely, EBS1, EBS2, and EBS3, existed at -2034 to $-2018 \mathrm{bp},-2006$ to $-2000 \mathrm{bp}$, and -1993 to $-1987 \mathrm{bp}$, respectively (Figure 4F). To explore these possible binding sites, we performed gel-shift assays using nuclear extracts from HUVECs. As shown in Figure 4G, a strong DNA complex was observed. The gel-shift experiment revealed that ox-LDL significantly increased DNA-protein complex formation. A 200-fold excess of wild-type cold competitive Ets-1 oligonucleotides (wt) eliminated the formation of the DNA/protein complex, while mutant cold competitive Ets-1 oligonucleotides (mut1, mut2, and mut3 for mutants of EBS1, EBS2, and EBS3, respectively) partially eliminated the formation of the DNA/protein complex (Figure 4F). As shown in Figure 4G, the EBS3 mutant nearly abolished the formation of the DNA-protein complex, whereas the EBS1 and EBS2 mutants did not. Furthermore, EBS2 was least able to eliminate the formation of the DNA/ protein complex (Figure 4G, lane 7). Therefore, the Ets-1 protein binds mainly to the EBS2 site of the DKK1 promoter. In the supershift lane, an obvious shift was found compared to the original position (Figure 4G, lane 9). This finding indicates that Ets-1 is a potential transcription factor for the DKK1 gene. To further confirm the above result, cultured 293T cells were transiently cotransfected with the $\mathrm{P} 0$ vector (or P0-del), the pRL-TK vector and PCDNA3.1-Ets-1 (P0 group, P0-del group). Further deletion of $6 \mathrm{bp}$ (from -2006 to $-2000 \mathrm{bp}$ ) resulted in a $50 \%$ decrease in promoter activity compared to that in the P0 group (Figure 4H), suggesting an important role of EBS2.

As expected, the crosslinked DNA-Ets-1 complexes immunoprecipitated with an Ets-1 antibody were detected by PCR amplification with primers spanning the region of the DKK1 promoter from -2080 to $-1894 \mathrm{bp}$. ChIP also revealed that, compared with the control, ox-LDL significantly increased the binding activity between the Ets-1 protein and the DKK1 promoter (Figure 4I-4L). The results indicated that Ets-1 increased the transcriptional activity of the DKK1 promoter.

\section{ox-LDL induced Ets-1, CBP, and c-jun binding to DKK1 promoter in HUVECs}

HUVECs were treated with ox-LDL for different durations. Western blot analysis showed that HUVECs treated with ox-LDL had higher c-jun and $\mathrm{c}$-fos expression than the $0 \mathrm{~h}$ group (Figure S2A and S2B). Immunofluorescence analysis showed that the nuclear translocation of c-jun, not c-fos, increased after ox-LDL treatment (Figure S2C). siRNA transfection downregulated the expression of c-jun and inhibited the ox-LDL-induced upregulation of DKK1 (Figure S2D and S2E). Cultured 293T cells were transiently cotransfected with the P0 and pRL-TK vectors and then transfected with NC siRNA or c-jun siRNA. A DLR assay showed that c-jun siRNA decreased DKK1 promoter activity in 293T cells (Figure S2F). 293T cells were cotransfected with P0, pRL-TK and PCDNA3.1 (control group) or cotransfected with P0-P9, pRL-TK and PCDNA3.1c-jun (group P0 -P9). A DLR assay showed that DKK1 promoter activity was increased in the P0 group and reduced from the P1 group to the P9 group (Figure S2G). The online prediction tools JASPAR and PROMO were used to identify possible loci and binding sites (-2029 to -2023bp) (Figure S2H). The results indicated that c-jun but not c-fos was involved in the regulation of DKK1 expression in ox-LDL-treated HUVECs.

Previous studies have shown that Ets-1 can recruit the transcriptional coactivator $\mathrm{CBP} / \mathrm{P} 300$ to target gene promoters and regulate gene expression. We showed that Ets-1 recruited the coactivator CBP to the DKK1 promoter. Pretreatment with a CBP/P300 inhibitor downregulated the expression of CBP/P300 and inhibited the ox-LDL-induced upregulation of DKK1 (Figure 5A). Cultured HUVECs were transiently cotransfected with the P0 and pRL-TK vectors and then treated with the $\mathrm{CBP} / \mathrm{P} 300$ inhibitor for $12 \mathrm{~h}$. A DLR assay was performed to determine the DKK1 promoter activity. The CBP/P300 inhibitor significantly decreased DKK1 promoter activity in $293 \mathrm{~T}$ cells compared with that in the control group 
(Figure 5B). The results indicated that CBP but not P300 was involved in the regulation of DKK1 expression in ox-LDL-treated HUVECs. Coimmunoprecipitation (co-IP) was performed after treatment with ox-LDL. The results showed that Ets-1 interacted with the endogenous CBP and with c-jun but not with P300 (Figure 5C). With a reverse co-IP assay, we showed that CBP also interacted with endogenous c-jun and Ets-1 (Figure 5D). After cotransfection with PCDNA3.1-c-jun and PCDNA3.1-Ets-1, DKK1 promoter activity was increased compared with that for co-transfection with PCDNA3.1-Ets-1 alone (Figure 5E). After cotransfection with PCDNA3.1c-jun and P0-del, the DKK1 promoter activity was decreased compared with that in the $\mathrm{P0}+$ PCDNA3.1-c-jun group (Figure 5F). Taken together, these observations indicated that Ets-1, CBP and c-jun might form a complex to regulate DKK1 activity coordinately.
Ets- 1 was a functional target of $\mathrm{miR}-33 \mathrm{a}-5 \mathrm{p}$ and miR33a-5p eliminated angiogenesis in ox-LDL-induced HUVECs

To examine potential miRNAs that negatively regulate DKK1 or Ets-1, a bioinformatics approach using multiple prediction algorithms (miRBase, PicTar, and TargetScan v6.1) was used to identify binding sites for miRNAs in the $3^{\prime}$-UTR of DKK1 and Ets-1. This analysis identified miR33a-5p as a potential regulator of DKK1 and Ets-1. ApoE-/- mice were given atherogenic chow for $0,4,8$ and 12 weeks, and miR33a-5p expression in the aortic artery was found to decrease with time (Figure 6A). HUVECs were treated with ox-LDL for different durations. qRT-PCR showed that ox-LDL downregulated the miR33a-5p expression in HUVECs compared with that in the $0 \mathrm{~h}$ group (Figure 6B). Dicer siRNA transfection upregulated the expression of DKK1 and Ets-1 (Figure S3A). Mimic transfection downregulated the expression of DKK1 and Ets-1, while inhibitor transfection upregulated the expression of DKK1 and Ets-1 (Figure 6C).
A
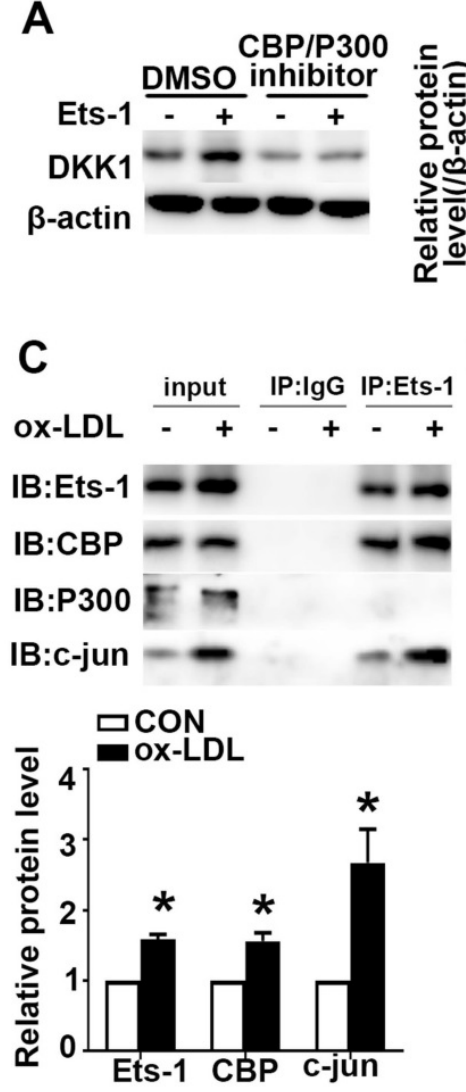

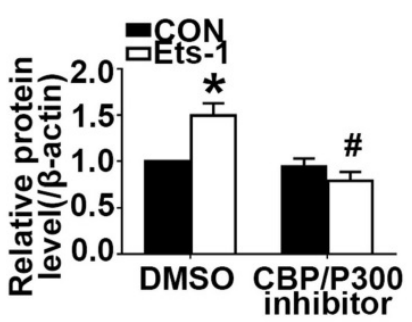

B
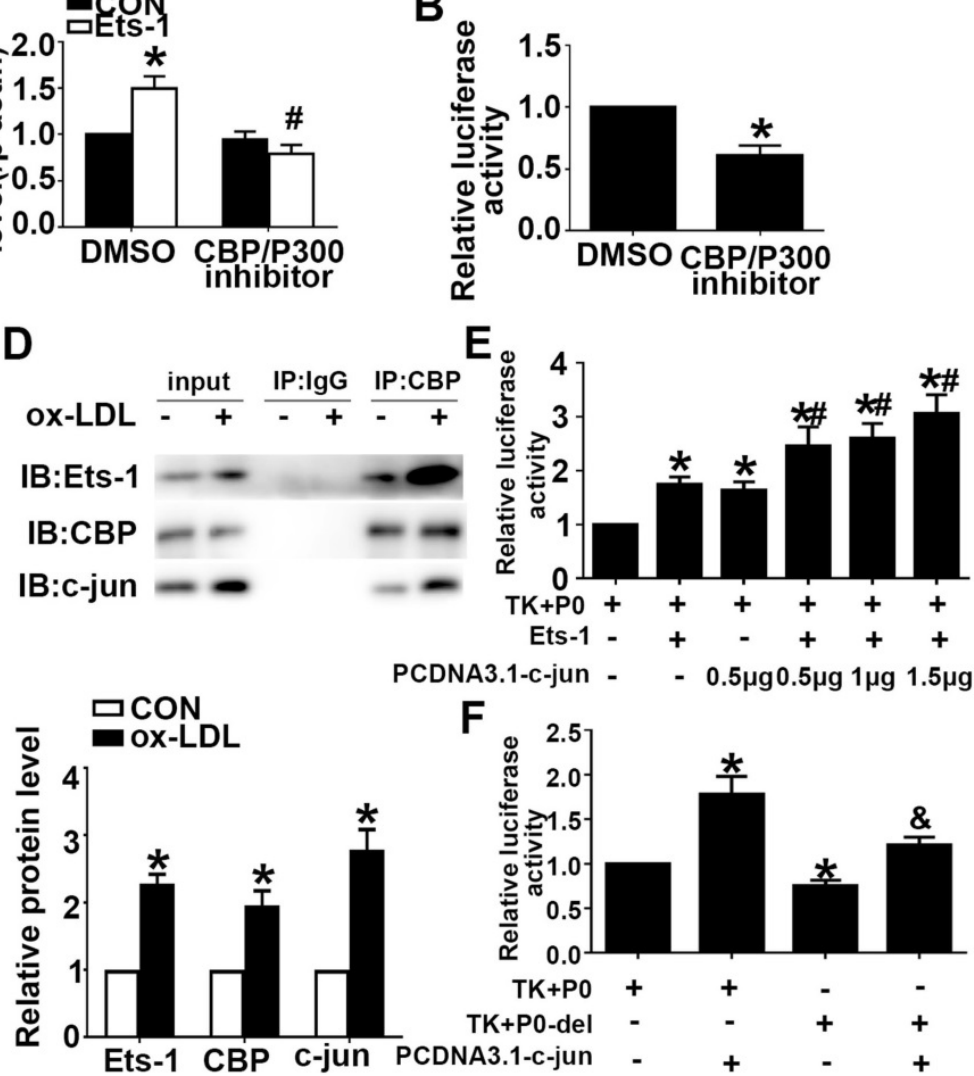

$\mathbf{F}$

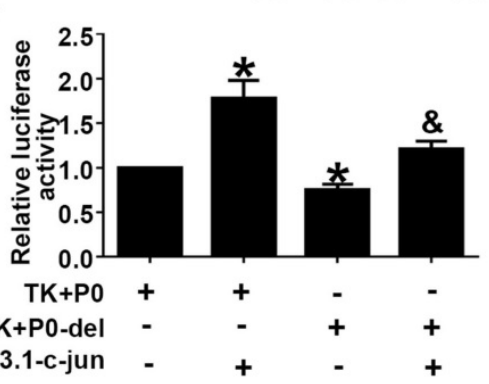

Figure 5. CBP interacts with Ets-1 and assists in activating the expression of DKK1. (A) HUVECs were pretreated with DMSO or a CBP/P300 inhibitor (25 $\mu$ M) for $1 \mathrm{~h}$ after transfection with lenti-Ets-1. Western blot for quantification of the protein levels of DKK $1 . n=6$. (B) HUVECs were pretreated with DMSO or CBP/P300 inhibitor (25 $\mu \mathrm{M})$ for $1 \mathrm{~h}$ before transfection with the $\mathrm{pGL3}$-full-length DKK1 promoter vector. The cells were collected, and the luciferase activities were analyzed. $\mathrm{n}=6$. (C) Cell lysates were immunoprecipitated by an anti-Ets-1 monoclonal antibody, and the precipitates were then immunoblotted with an anti-CBP, anti-P300 or anti-c-jun antibody. $n=3$. (D) Cell lysates were immunoprecipitated by an anti-CBP monoclonal antibody, and the precipitates were then immunoblotted with anti-Ets-1 or anti-c-jun antibody. $n=3$. (E) $293 T$ cells were cotransfected with PCDNA3.1-c-jun and PCDNA3.1-Ets-1. The luciferase activities were analyzed. $n=6$. (F) 293T cells were co-transfected with PCDNA3.1-c-jun and $P 0$-del (or P0). The luciferase activities were analyzed. $n=6$. The data are presented as the mean $\pm S E M$. $* P<0.05$ vs. the control group, $\# P<0.05$ vs. the $P 0+E t s-1$ group, $\& P<0.05$ vs. the $\mathrm{PO}+\mathrm{c}$-jun group. 

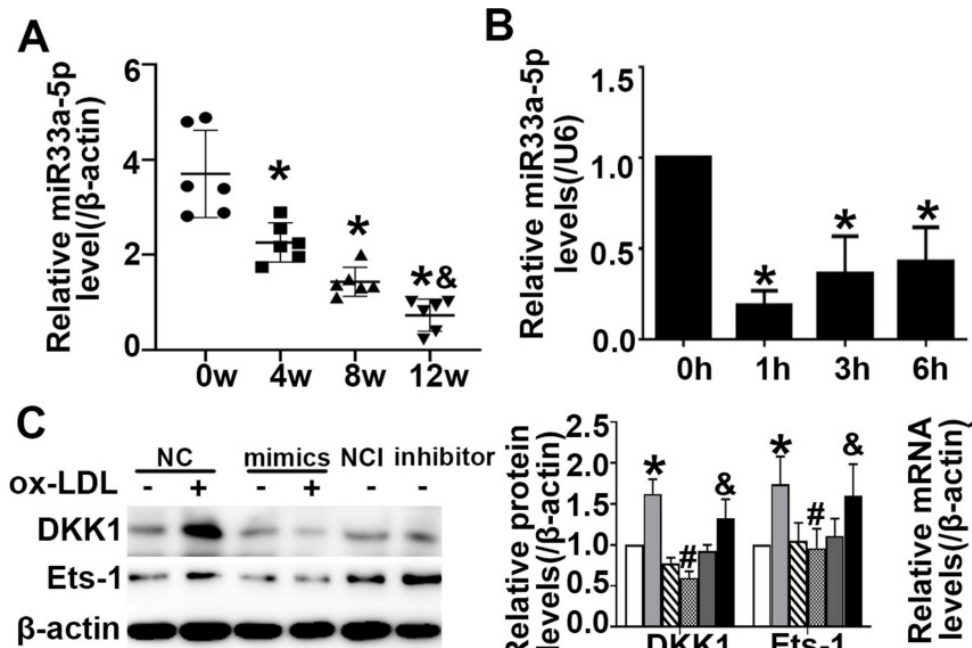

D

Pmir-GLO-Ets-1 3'-UTR

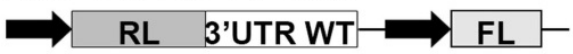

Pmir-GLO-Ets-1 mutant 3'-UTR
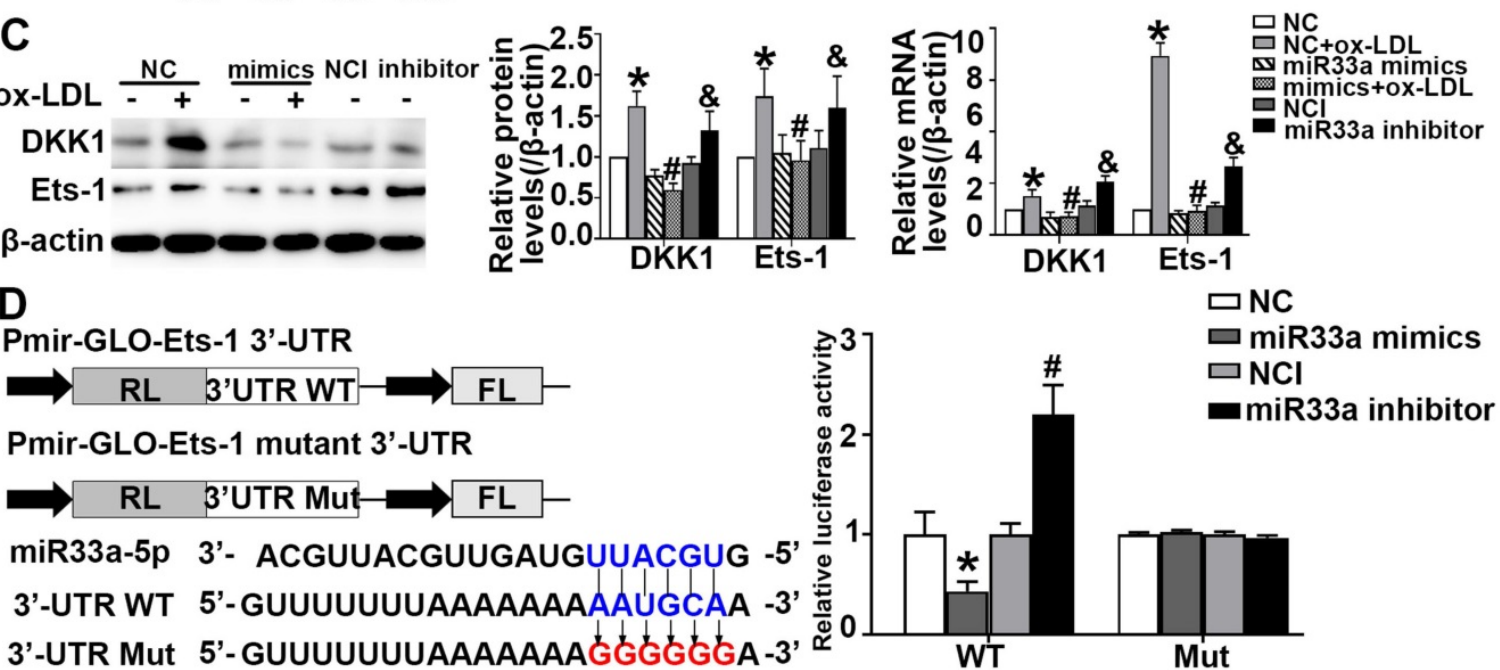

E NC NC+ox-LDL miR33a mimics +ox-LDL ${ }^{\text {miR } 33 a \text { mimics }}$

$$
\begin{aligned}
& \square \mathrm{miR33a} \text { mimics } \\
& \square \mathrm{NCl} \\
& \square_{\mathrm{miR} 33 \mathrm{a}} \text { inhibitor }
\end{aligned}
$$

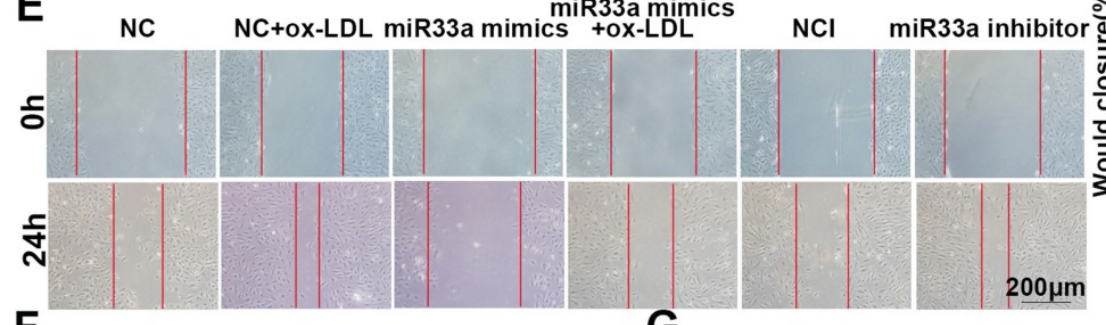

$\mathbf{F}$
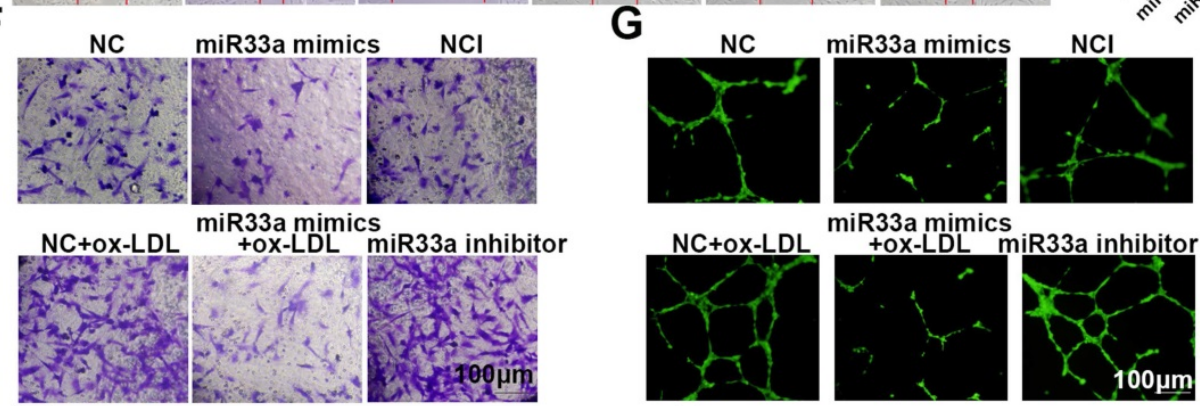

miR33a mimics
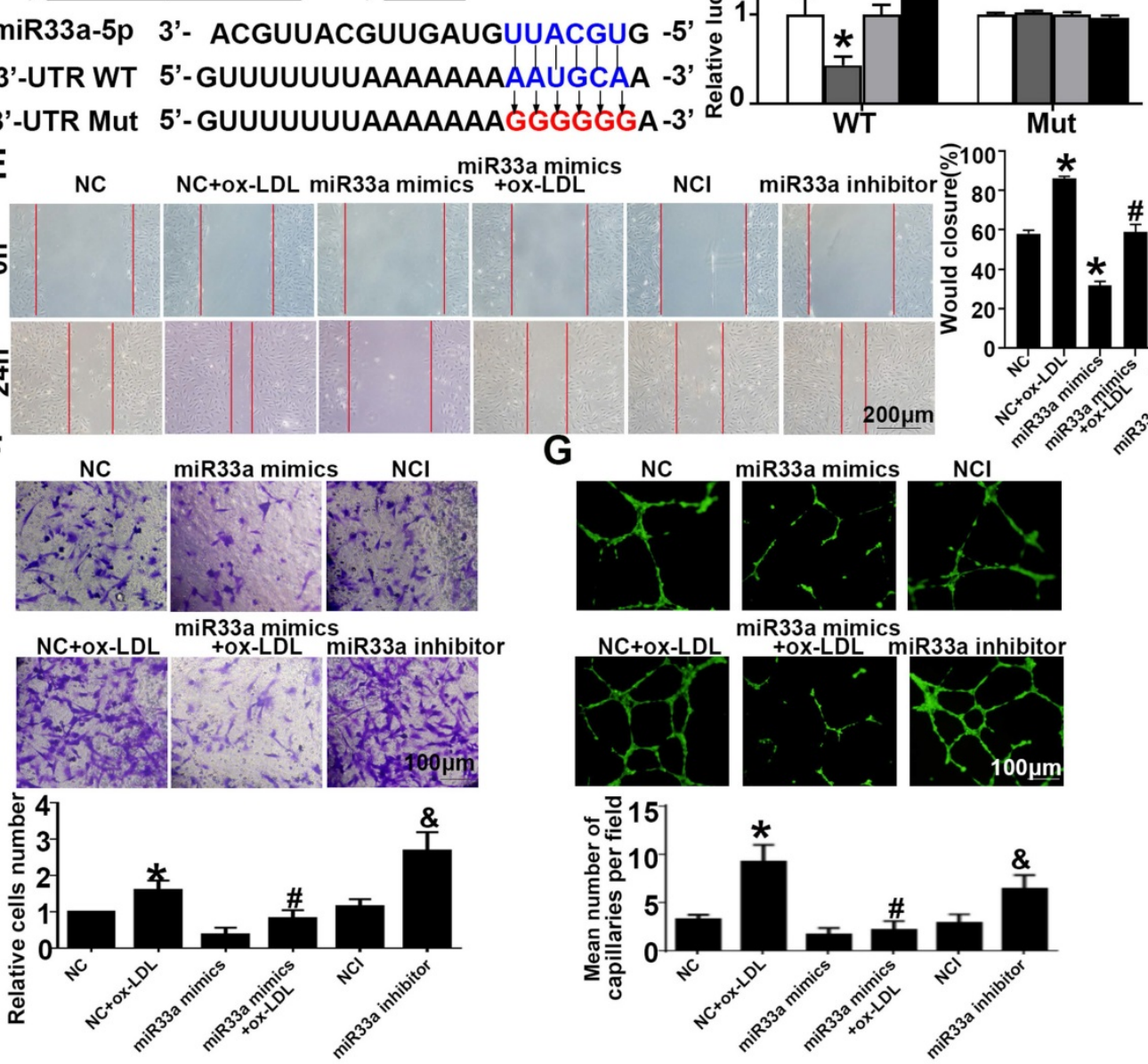

Figure 6. miR33a-5p promotes the migration and angiogenesis of HUVECs by directly targeting and degrading Ets-1. (A) miR33a-5p expression in the aortic artery in ApoE-/- mice treated with atherogenic chow for 0, 4, 8 and 12 weeks as determined by qRT-PCR. (B) PCR analysis of miR33a-5p in HUVECs treated with ox-LDL (150 $\mathrm{\mu g} / \mathrm{ml})$ for various lengths of time $(0 \mathrm{~h}, 1 \mathrm{~h}, 3 \mathrm{~h}$, and $6 \mathrm{~h}$ ). (C) Western blot analysis of Ets-1 and DKK1 after miR33a-5p mimic or miR33a-5p inhibitor transfection in ox-LDL-treated HUVECs. (D) Possible binding sites for miR33a-5p in the Ets-1 3'-UTR, as predicted. A miR target reporter luciferase assay was performed after the miR33a-5p mimic and inhibitor were delivered to $293 \mathrm{~T}$ cells. The results were normalized to data obtained from an assay with Renilla luciferase. (E) Representative images and quantification of cell migration in Transwell assays in miR33a-5p mimic-or miR33a-5p inhibitor-transfected HUVECs. The cell count on the bottom of the Transwell shown here. Bars indicate $200 \mu \mathrm{m}$. (F) Representative images and quantification of cell migration in the in vitro scratch wound assay (relative to $0 \mathrm{~h}$ ) from $0 \mathrm{~h}$ and $24 \mathrm{~h}$ post-wounding in miR33a-5p mimic or inhibitor-transfected HUVECs. Bars indicate $100 \mu \mathrm{m}$. (G) Representative image and quantification of the tube length (\% of control) of the miR33a-5p mimic or inhibitor-transfected HUVECs. Bars indicate $100 \mu \mathrm{m}$. The data are presented as the mean $\pm \mathrm{SEM}$. $\mathrm{n}=6$. $* P<0.05$ vs. the NC group; \#P<0.05 vs. NC+ ox-LDL; \&P <0.05 vs. NCl. 
Transient cotransfection of miR33a-5p mimics with Ets-1 3'-UTR luciferase reporter plasmids resulted in significant repression of luciferase reporter gene expression in 293T cells, whereas cotransfection of $293 \mathrm{~T}$ cells with NC miRNA or the mutants did not have any effect on luciferase expression. Transient cotransfection of the miR33a-5p inhibitor with Ets-1 3 '-UTR luciferase reporter plasmids gave the opposite result (Figure 6D). The results indicated that miR33a-5p was involved in the regulation of Ets-1 and DKK1 and directly bound to the 3 '-UTR of Ets-1. Transient cotransfection of miR33a-5p mimics with DKK1 3'-UTR luciferase reporter plasmids did not have any effect on the expression of luciferase in 293 cells, and cotransfection of 293T cells with NC miRNA or the mutants also did not have any effect on the expression of luciferase. Transient cotransfection of the miR33a-5p inhibitor with DKK1 3'-UTR luciferase reporter plasmids gave the same result (Figure S3B). The results indicated that miR33a-5p was involved in the regulation of Ets-1 and DKK1 and bound directly to the 3'-UTR of Ets-1.

Compared with the NC+ox-LDL group, the miR33a-5p mimics+ox-LDL group showed less migration and tube formation (Figure 6E-6G). Compared with the NCI group, the miR33a-5p inhibitor group showed more migration and tube formation (Figure 6E-6G). The results indicated that miR33a-5p eliminated migration and angiogenesis of ox-LDL-induced HUVECs.

\section{miR33a-5p exhibited antiangiogenic effect in ox-LDL-induced HUVECs via degrading Ets-1/DKK 1}

Compared with the miR33a-5p mimics group, the miR33a-5p mimics+lenti-Ets-1 group showed elevated migration and tube formation (Figure 7A-7C). Compared with the miR33a-5p inhibitor group, the miR33a-5p inhibitor +Ets-1 siRNA group showed less migration and tube formation (Figure S4A-S4C). The results suggest that miR33a-5p leads to ox-LDL-induced migration and tube formation by inhibiting Ets-1 in ECs.

Compared with the NC group, the NC+lentiEts-1 group showed more migration and tube formation. Compared with the NC+lenti-Ets-1 group, the DKK1 siRNA+lenti-Ets-1 group showed less migration (Figure 7D-AF). These results suggest that Ets- 1 causes the migration of ECs and tube formation by inducing DKK1.

Next, compared with the lenti-Ets-1 group, the CBP/P300 inhibitor+lenti-Ets-1 group showed less migration and tube formation (Figure S4D-S4F). This result suggests that $\mathrm{CBP} / \mathrm{P} 300$ participates in the Ets-1-induced migration and tube formation.

\section{Discussion}

In this study, we found that DKK1, a tumorigenesis associated molecule, promoted angiogenesis of carotid atherosclerotic plaques in high fat fed ApoE-/- mice. ox-LDL stimulates the secretion of TNF-a from macrophages and cytokines from ECs, indicates the starting point of atherosclerosis. To further explore the findings in vivo, ox-LDL was used to stimulate HUVECs in vitro in our study [21]. We demonstrated that the high expression of DKK1 under ox-LDL stimulus increased the migration and angiogenesis in HUVECs via CKAP4/PI3K pathway. Data from Western blotting, real-time RT-PCR, electrophoretic mobility shift assay (EMSA) and ChIP revealed that upstream nuclear transcription factor Ets- 1 could bind to the DKK1 promoter region, form a complex with CBP and c-jun, increase the transcriptional activity of the DKK1 promoter and promote DKK1 expression in the ox-LDL-treated HUVECs. Meanwhile, miR33a-5p was found to directly target the 3 '-UTR of Ets-1 to regulate the expression of Ets-1 and DKK1. To the best of our knowledge, this is the first study to reveal the role and the underlying mechanisms of DKK1 in angiogenesis under the treatment of ox-LDL in HUVECs.

Angiogenesis, also named neovascularization, refers to the growth of new blood vessels that sprout from existing blood vessels. It's a complex process involved in differentiation, proliferation, migration and maturation of endothelial cells. Studies showed that angiogenesis intra atherosclerotic lesions plays vital roles in plaque growth and instability [22], and is of vital importance in plaque progression, plaque destabilization and thromboembolic events. Several angiogenesis-related genes, like VEGF, platelet-derived growth factor (PDGF) and tumor growth factor- $\beta$ (TGF- $\beta$ ) in endothelial cells were demonstrated to be induced by atherosclerotic risk factors, such as oxidative stress, inflammatory factors and mechanical forces [23,24]. The VEGF family which consists of five closely related members, namely VEGF-A, B, C, D and placental growth factor, played important roles in angiogenesis. The release of VEGF-A and activation of VEGFR-2, a receptor for VEGF-A contributes significantly to promote intra atherosclerotic angiogenesis $[25,26]$. There were also studies showed that non-coding RNA regulated endothelial proliferation, migration and tube formation, and ultimately affected angiogenesis [27]. Researchers have explored to target the intra-plaque angiogenesis through inhibition of vascular endothelial growth factor signaling, glycolytic flux and fatty acid oxidation [28-30]. However, although anti-angiogenesis therapy has widely used in cancer treatment, studies on the pharmacological inhibition 
of this phenomenon in AS are still scare [31]. In our study, we found that DKK1 was highly expressed and promoted the angiogenesis via increasing the expression of VEGF-A, VEGFR-2, MMP-2 and MMP-9 in the atherosclerotic plaques. CD31 is a marker of angiogenesis [32]. In vivo, we detected the expression of CD31 to assess angiogenesis. The in vitro study in the ox-LDL-treated HUVECs also vertified these findings, which may provide a new potential intervention target for the anti-angiogenesis therapy in AS.
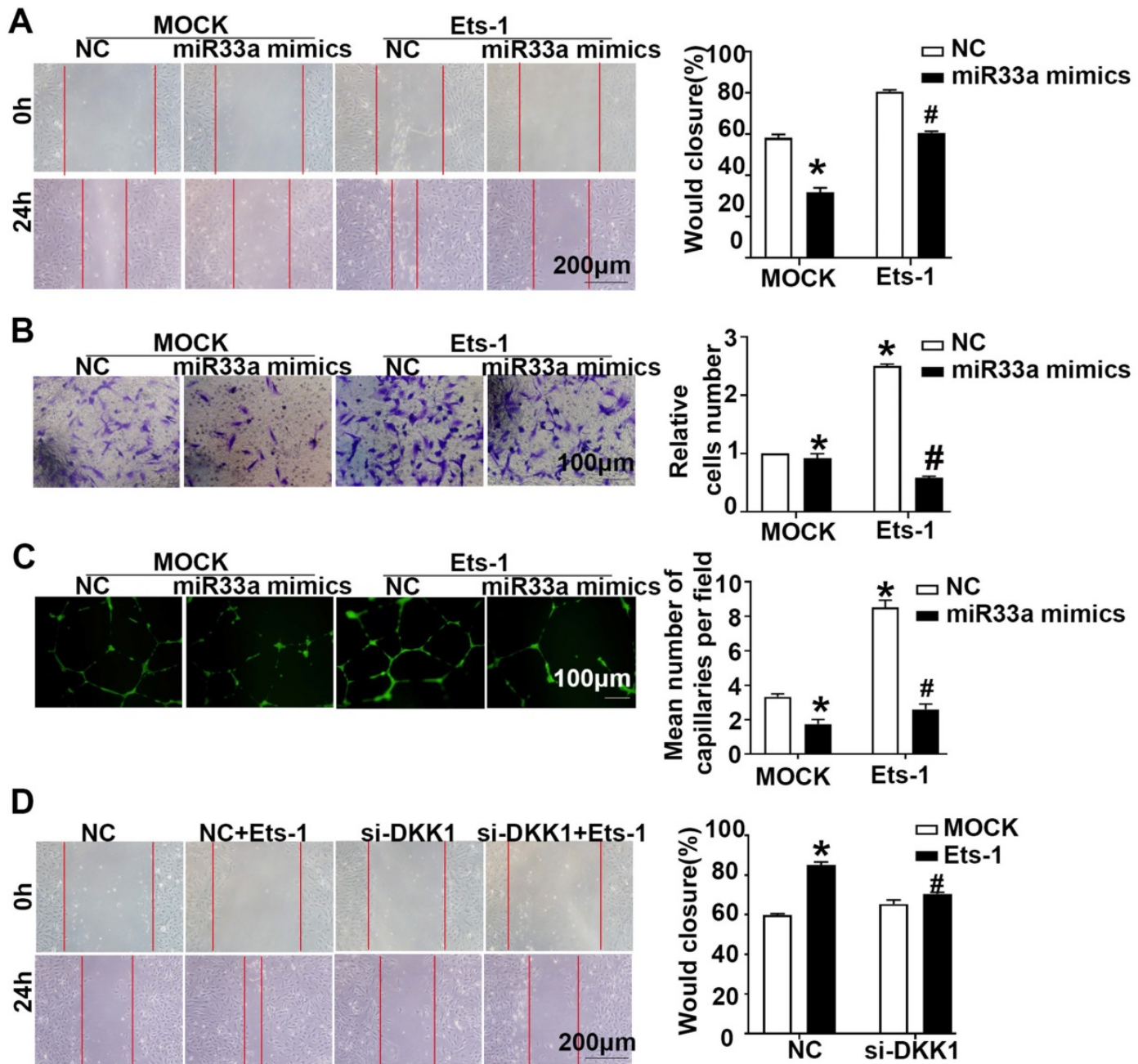

$\mathbf{E}$
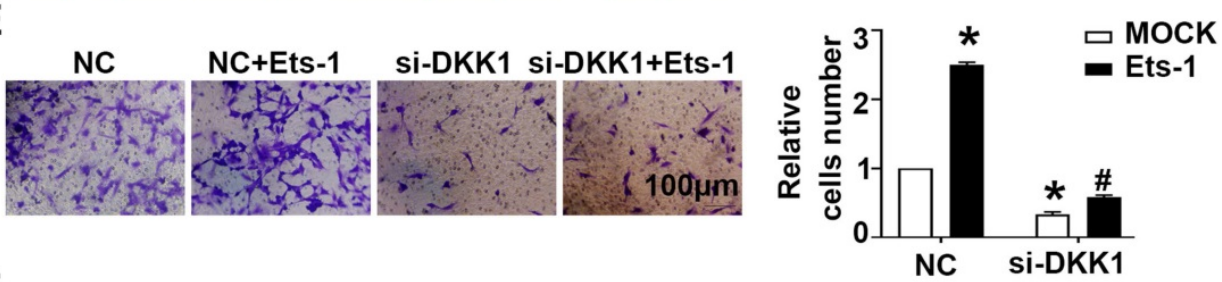

$\mathbf{F}$
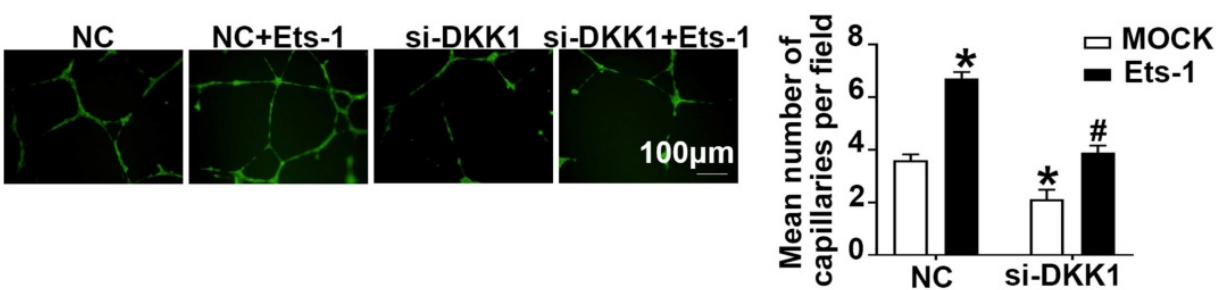

Figure 7. miR33a-5p exhibits antiangiogenic effects in ox-LDL induced HUVECs by degrading Ets-1/DKK1. (A, D) Representative images and quantification of cell migration in the in vitro scratch wound assay (relative to 0 h) were obtained at 0 h and $24 \mathrm{~h}$ post-wounding, bars indicate $200 \mu \mathrm{m}$ : (A) in miR33a mimic-transfected $\mathrm{HUVEC}$ before transfected lenti-Ets-1; (D) in DKK1 siRNA-transfected HUVECs after transfection with lenti-Ets-1. (B, E) Representative images and quantification of cell migration in Transwell assays, bars indicate $100 \mu \mathrm{m}$ : (B) miR33a-5p mimic-transfected HUVECs before transfection with lenti-Ets-1; (E) DKK1 siRNA-transfected HUVECs after transfection with lenti-Ets-1. The cell counts on the bottom of the Transwell are shown here. (C, F) Representative image and quantification of the tube length (\% of control), bars indicate $100 \mu \mathrm{m}:(\mathrm{C})$ in the miR33a-5p mimic-transfected HUVECs before transfection with lenti-Ets-1; (F) in the DKK1 siRNA-transfected HUVECs after transfection with lenti-Ets-1. The data are presented as the mean \pm SEM. $n=6$. $* P<0.05$ vs. the NC group; $\# P<0.05$ vs. miR33a-5p mimic or Ets-1. NC, Negative control; Ets-1, lentivirus Ets-1. 
DKK1, a secretory glycoprotein of the DKK family, had been found to play vital roles both in cancers and AS. At present, DKK1 has been developed as a serological marker for the diagnosis and prognosis evaluation of several cancers, and also a new target for cancer treatment. In the registered clinical trials, we found DKK1 antibody DKN-01 had been entered into clinical phase I or phase II trial in advanced biliary tract cancer [7], advanced liver cancer, cholangiocarcinoma, gastric cancer and other tumors, and completed a promising study in gastric/gastroesophageal junction cancer [3,33]. Another DKK1 antibody BHQ880 had completed the clinical phase II experiment for multiple myeloma in year 2020. Oncological treatment may increase the morbidity and mortality of cardiovascular diseases (CVDs) as a side effect [4]. Our previous studies have revealed that DKK1 could promote endothelial apoptosis [5], destroy the tight junctions of endothelium [34], disturb the lipid metabolism [35] and lead to the development of AS. Anti-DKK1 neutralizing antibodies have shown promise and might be beneficial for the treatment of CVDs [7]. Recent studies reported that angiogenesis was a link between atherosclerosis and tumorigenesis [36]. Investigating the role of DKK1 in neovascularization will benefit both for the cancer and CVD treatment at the same time. Therefore, we further revealed the role and underlying mechanisms of DKK1 in AS from the perspective of angiogenesis in this study. Our findings showed that DKK1 promoted the migration and angiogenesis via up-regulating the angiogenesisrelated molecules by activation of CKAP4/PI3K pathway, which indicated that anti-DKK1 therapy may prevent tumor and meanwhile reduce unstable plaques [37]. Furthermore, we explored the upstream regulation mechanism of DKK1. Previous studies have revealed several mechanisms upstream of DKK1, including the aspects of histone modification, transcription, posttranscriptional modification and posttranslational modification (phosphorylation, glycosylation). It was reported that transcription factors (YAP, $\beta$-catenin, etc) binded to the DKK1 promoter region directly to activate its transcription $[10,38,39]$. In this study, we found that nuclear transcription factors, c-jun and Ets-1, could bind to the DKK1 promoter region and activate DKK1 promoter. Using DLR, EMSA and ChIP experiments, we also clarified that Ets-1 binded to the -2006 to $-2000 \mathrm{bp}$ region of the DKK1 promoter and c-jun binded to the -2029 to $-2023 b p$ region of the DKK1 promoter. Ets-1, a member of the Ets family of transcription factors, plays important roles in cellular proliferation, migration, vascular remodeling and apoptosis. Hypoxia, inflammatory factors and VEGF upregulate the expression of Ets-1 in ECs [14]. Previous studies showed that Ets-1 performed multiple functions in ECs: (1) Ets-1 directly regulated several vascular genes, such as Flt1, Tek Kdr, Angpt2, Nrp1, Vwf, Pecam 1 and Cdh5, to promote angiogenesis [13], and (2) Ets-1 directly upregulated MMPs and $\beta 3$ integrin to promote migration $[15,16]$. Feng, et al found that Ets-1 participated in inflammation induced carotid artery endoluminal vascular injury and promoted AS [40]. We found that the expression of Ets-1 was significantly upregulated by ox-LDL in HUVECs, Ets-1 siRNA decreased DKK1 expression and relieved the ox-LDL-induced migration of ECs and angiogenesis, indicating that ox-LDL may cause changes through the Ets-1/DKK1 regulation of EC function. These findings further deepen our understanding of the regulatory mechanism of DKK1.

In addition, we also found that c-jun, one of AP-1 performs, upregulated the expression of DKK1 via increasing the promoter activity of it under the stimulus of ox-LDL. Previous studies have shown that c-jun promoted the expression of VEGF and induced angiogenesis [41]. The transcription coactivators CBP and P300, which consist of four transcription factor binding domains (TADs) to recruit transcription factors, change chromatin superstructures, and activate acetylation [42], could promote the activation of a variety of transcription factors [43], such as c-jun (which binds to the CREB binding domain) [44], c-fos (which binds to third zinc finger domains) [45], and Ets-1 (which binds to first zinc finger domains) [46]. The bindings of Ets-1, AP-1 [14,47], and the coactivator $\mathrm{CBP} / \mathrm{P} 300$ promote the connection of these two transcription factors [13]. CBP/P300 is also involved in the acetylation of the DKK1 promoter in breast cancer [48]. In our studies, we found protein binding among CBP, Ets-1 and c-jun. Ets-1 increased the transcriptional activity of DKK1 with the aid of c-jun and CBP. The effect of Ets-1 on migration and angiogenesis was reversed by a CBP/P300 inhibitor. The results indicated that $\mathrm{CBP}$ assisted in the effects of Ets-1/DKK1 on EC function.

MiRNAs are small (19-23 nucleotides) non-coding RNAs which regulate the target genes by binding to the $3^{\prime}$ untranslated region (UTR) of mRNA post-transcriptionally. Studies have shown that miRNAs play vital roles in the development of AS and the pathological processes of ECs. Previous studies reported that the expression of Ets-1 in ECs can be inhibited by miR155 [49], miR-199a-5p [13], the miR-200 family [50] and miR-221/222 [49]. In addition, miR-217 [51], miR152 [11], miR-376a [52] and other miRNAs decreased the expression of DKK1 by inhibiting translation or decreasing mRNA stability in different diseases. A previous study found 
that DKK1 is regulated by miR217, miR33a, miR33b, miR103a, miR93 and miR106a in diabetic cardiomyopathy [20]. In this study, we further clarified the possible miRNAs in the regulation of Ets-1 and DKK1. Micro.org, STARBASE, TargetScan and other microRNA programs predicted that both Ets-1 and DKK1 had probable binding regions with the miR33a-5p (3'-UTR) binding sites. In a DLR assay, miR33a-5p bound to the Ets-1 3'-UTR but not the DKK1 3'-UTR, which suggested that miR33a-5p may inhibit the expression of Ets-1 and thus play a role in the regulation of DKK1. At present, there are few studies on miR33a. There are two subtypes of miR33 in humans, miR33a and miR33b [53]. Human miR33a and the only mouse homologue (miR-33) are located at intron 16 of SREBP-2. It was reported to regulate the chemoresistance, proliferation, invasion and angiogenesis in tumor [54]. MiR33a was also closely related to the lipid metabolism (inhibition of ABCA1 and cholesterol efflux, inhibition of HDL production) [18]. Horie $\mathrm{T}$, et al found that miR-33 deficiency reduced atherosclerotic plaque size and lipid content suggesting that miR-33 inhibition may prevent atherosclerosis progression [53]. Talepoor, et al found that ECs exhibit a protective effect and inhibit miR-33a expression in monocytes, when cocultured with monocytes. The result indicated miR33a might play a protective role in the formation of atherosclerosis [55]. As well, the expression of miR33a-5p decreased over time in the high fat fed ApoE-/- mice in our study, which indicating down-regulation of miR33a-5p in the development of atherosclerosis. However, whether miR33a affected plaque development by affecting endothelial cell function needs further proof. The effect of EC specific miR33a on atherosclerosis is the limitation of this study. In our study, we further explored whether miR-33a exerted great effects in the angiogenesis in the ox-LDL stimulated HUVECs and found that miR33a-5p directly bound to Ets-1 3'-UTR, decreased the expression of Ets-1 and DKK1, and attenuated the migration of endothelial cells and angiogenesis.

In conclusion, DKK1 promoted angiogenesis intra atherosclerotic plaques. DKK1 upregulated migration and angiogenesis of HUVECs via the CKAP4/PI3K pathway, not via $W n t / \beta$-catenin pathway. miR-33a/Ets-1/DKK1 axis exerted important effects on the migration and angiogenesis in HUVECs under the stimulus of ox-LDL. The protein binding complex by CBP, c-jun and Ets-1 facilitated this process. Deep exploration of the underlying mechanisms in the angiogenesis in atherosclerosis is essential for the designing novel therapeutic targets, and is also important for the recognition of the cardiovascular side effects in the process of related anti-tumor treatment in future.

\section{Abbreviations}

AS: atherosclerosis; ChIP: chromatin immunoprecipitation; CKAP4: cytoskeleton-associated protein 4; co-IP : coimmunoprecipitation; CRDs: cysteine rich domains; CVDs: cardiovascular diseases; DKK1: Dickkopf1; EC: endothelial cell; EdU: Five-ethynyl2'-deoxyuridine; EMSA: electrophoretic mobility shift assay; DLR : dual-luciferase reporter assay; HUVECs : human umbilical vein endothelial cells; IHC: Immunohistochemical analysis; miRNA: microRNA; NC: negative control; NS: normal saline; qRT-PCR: realtime quantitative polymerase chain reaction; RCA: right carotid artery; siRNA: small interfering RNA; 3'-UTR: 3'-untranslated region.

\section{Supplementary Material}

Supplementary figures and tables. http://www.ijbs.com/v17p4122s1.pdf

\section{Acknowledgements}

\section{Funding}

This work was supported by funding from the National Natural Science Foundation of China (82000419, 81770439, 81700387 and 81900621) and the National Research Program of China (2016 YFC1300302).

\section{Author Contributions}

MXD, Meng Z, CZ and Mei $Z$ designed the experiments and wrote the manuscript. MXD, YZ and WJC performed the major experiments, including RT-qPCR, ELISA, immunoblots, luciferase assay, and animal experiments. MXD, RYZ and XLL analyzed the experiments. MXD, MML and Mei $Z$ was of immense help in the modification of the manuscript. All authors read and approved the final manuscript.

\section{Competing Interests}

The authors have declared that no competing interest exists.

\section{References}

1. Kagey $\mathrm{MH}, \mathrm{He} X$. Rationale for targeting the wnt signaling modulator DKK1 for oncology. Br J Pharmacol. 2017; 174:4637-4650.

2. Wall JA, Klempner SJ, Arend RC. The anti-DKK1 antibody DKN-01 as an immunomodulatory combination partner for the treatment of cancer. Expert Opin Investig Drugs. 2020; 29:639-644.

3. Haas MS, Kagey MH, Heath H, Schuerpf F, Rottman JB, and Newman W. mDKN-01, a Novel Anti-DKK1 mAb, Enhances Innate Immune Responses in the Tumor Microenvironment. Mol Cancer Res. 2021; 19:717-725.

4. Zamorano JL, Lancellotti P, Muñoz DR, Aboyans V, Asteggiano R, Galderisi M, et al. 2016 ESC Position Paper on cancer treatments and cardiovascular toxicity developed under the auspices of the ESC Committee for Practice Guidelines: The Task Force for cancertreatments and cardiovascular toxicity of the European Society of Cardiology (ESC). Eur J Heart Fail. 2017; 19:9-42. 
5. Di M, Wang L, Li M, Zhang Y, Liu X, Zeng R, et al. Dickkopf1 destabilizes atherosclerotic plaques and promotes plaque formation by inducing apoptosis of endothelial cells through activation of ER stress. Cell Death Dis. 2017; 8:e2917.

6. Baetta R, Banfi C. Dkk (Dickkopf) proteins. Arterioscler Thromb Vasc Biol. 2019; 39:1330-1342.

7. Goyal L, Sirard C, Schrag M, Kagey MH, Eads JR, Stein S, et al. Phase I and Biomarker Study of the Wnt Pathway Modulator DKN-01 in Combination with Gemcitabine/Cisplatin in Advanced Biliary Tract Cancer. Clin Cancer Res. 2020; 26:6158-6167.

8. Hergenreider E, Heydt S, Tréguer K, Boettger T, Horrevoets AJ, Zeiher $\mathrm{AM}$, et al. Atheroprotective communication between endothelial cells and smooth muscle cells through miRNAs. Nat Cell Biol. 2012; 14:249-56.

9. Ming Z, Wang Y, Gong A, Zhang X, Li M, Chen T, et al. Attenuation of Intestinal Epithelial Cell Migration During Cryptosporidium parvum Infection Involves Parasite Cdg7_FLc_1030 RNA-Mediated Induction and Release of Dickkopf-1. The Journal of infectious diseases. 2018; 218:1336-1347.

10. Ntini E, Louloupi A, Liz J, Muino JM, Marsico A, Ørom UAV. Long ncRNA A-ROD activates its target gene DKK1 at its release from chromatin. Nat Commun. 2018; 9:1636.

11. Zhao X, Sun SC, Xu JT, Luo Y, Xin Y, Wang YB. MicroRNA-152 inhibits cell proliferation of osteosarcoma by directly targeting Wnt/beta-catenin signaling pathway in a DKK1-dependent manner. Oncol Rep. 2018; 40:767-774.

12. Haniu M, Horan T, Spahr C, Hui J, Fan W, Chen C, et al. Human Dickkopf-1 (huDKK1) protein: Characterization of glycosylation and determination of disulfide linkages in the two cysteine-rich domains. Protein Sci. 2011; 20:1802-1813.

13. Sizemore GM, Pitarresi JR, Balakrishnan S, Ostrowski MC. The ETS family of oncogenic transcription factors in solid tumours. Nat Rev Cancer. 2017; 17:337-351

14. Meadows SM, Myers CT, Krieg PA. Regulation of endothelial cell development by ETS transcription factors. Seminars in cell \& developmental biology. 2011; 22:976-984

15. Oettgen P. Regulation of vascular inflammation and remodeling by ETS factors. Circ Res. 2006; 99:1159-1166.

16. Teruyama K, Abe M, Nakano T, Iwasaka-Yagi C, Takahashi S, Yamada S, et al. Role of transcription factor Ets-1 in the apoptosis of human vascular endothelial cells. J Cell Physiol. 2001; 188: 243-52.

17. Laffont B, Rayner KJ. MicroRNAs in the pathobiology and therapy of atherosclerosis. Can J Cardiol. 2017; 33:313-324.

18. Rayner KJ, Sheedy FJ, Esau CC, Hussain FN, Temel RE, Parathath S, et al. Antagonism of miR-33 in mice promotes reverse cholesterol transport and regression of atherosclerosis. J Clin Invest. 2011; 121:2921-2931.

19. Zhu C, Zhao Y, Zhang Z, Ni Y, Li X, Yong H. MicroRNA-33a inhibits lung cancer cell proliferation and invasion by regulating the expression of catenin. Molecular medicine reports. 2015; 11:3647-3651.

20. Ling S, Birnbaum Y, Nanhwan MK, Thomas B, Bajaj M, Li Y, et al. Dickkopf-1 (DKK1) phosphatase and tensin homolog on chromosome 10 (PTEN) crosstalk via microRNA interference in the diabetic heart. Basic Res Cardiol. 2013; 108:352.

21. Liang $\mathrm{H}$, Chen $\mathrm{M}$, Qi $\mathrm{F}$, Shi $\mathrm{L}$, Duan $\mathrm{Z}$, Yang $\mathrm{R}$, et al. The proatherosclerotic function of indoleamine 2, 3-dioxygenase 1 in the developmental stage of atherosclerosis. Signal Transduct Target Ther. 2019; 4:23.

22. Camaré $C$, Pucelle $M$, Nègre-Salvayre A, Salvayre R. Angiogenesis in the atherosclerotic plaque. Redox Biol. 2017; 12:18-34.

23. Yancopoulos GD, Davis S, Gale NW, Rudge JS, Wiegand SJ, Holash J. Vascular-specific growth factors and blood vessel formation. Nature. 2000; 407:242-248.

24. Zanotelli MR, Reinhart-King CA. Mechanical Forces in Tumor Angiogenesis. Adv Exp Med Biol. 2018; 1092:91-112.

25. Simons M, Gordon E, Claesson-Welsh L. Mechanisms and regulation of endothelial VEGF receptor signalling. Nat Rev Mol Cell Biol. 2016; 17:611-625.

26. Inoue $M$, Itoh $H$, Ueda $M$, Naruko $T$, Kojima $A$, Komatsu $R$, et al. Vascular endothelial growth factor (VEGF) expression in human coronary atherosclerotic lesions: possible pathophysiological significance of VEGF in progression of atherosclerosis. Circulation. 1998; 98:2108-2116

27. Zhao Z, Sun W, Guo Z, Zhang J, Yu H, Liu B. Mechanisms of lncRNA/ microRNA interactions in angiogenesis. Life Sci. 2020; 254:116900.

28. Stefanadis C, Toutouzas K, Stefanadi E, Lazaris A, Patsouris E, Kipshidze N. Inhibition of plaque neovascularization and intimal hyperplasia by specific targeting vascular endothelial growth factor with bevacizumab-eluting stent: An experimental study. Atherosclerosis. 2007; 195:269-276.
29. Perrotta P, Emini Veseli B, Van der Veken B, Roth L, Martinet W, De Meyer GRY. Pharmacological strategies to inhibit intra-plaque angiogenesis in atherosclerosis. Vascul Pharmacol. 2019; 112:72-78.

30. Schoors S, Bruning U, Missiaen R, Queiroz KC, Borgers G, Elia I, et al. Fatty acid carbon is essential for dNTP synthesis in endothelial cells. Nature. 2015; 520:192-197.

31. Dorst DCHV, Doorn LV, Mirabito Colafella KM, Manintveld OC, Hassing HC, Danser AHJ, et al. Cardiovascular toxicity of angiogenesis inhibitors and immune checkpoint inhibitors: synergistic anti-tumour effects at the cost of increased cardiovascular risk? Clin Sci (Lond). 2021; 135:1649-1668.

32. Liao Y, Wang C, Yang Z, Liu W, Yuan Y, Li K, et al. Dysregulated Sp1/ miR-130b-3p/HOXA5 axis contributes to tumor angiogenesis and progression of hepatocellular carcinoma. Theranostics. 2020; 10:5209-5224.

33. Mazon M, Masi D and Carreau M. Modulating Dickkopf-1: A Strategy to Monitor or Treat Cancer? Cancers (Basel). 2016; 8:62.

34. Li M, Liu X, Zhang Y, Di M, Wang H, Wang L, et al. Upregulation of Dickkopf1 by oscillatory shear stress accelerates atherogenesis. J Mol Med (Berl). 2016; 94:431-441.

35. Zhang Y, Ge C, Wang L, Liu X, Chen Y, Li M, et al. Induction of DKK1 by ox-LDL negatively regulates intracellular lipid accumulation in macrophages. FEBS Lett. 2015; 589:52-58.

36. Jiang J, Yan M, Mehta JL, Hu C. Angiogenesis is a Link Between Atherosclerosis and Tumorigenesis: Role of LOX-1. Cardiovasc Drugs Ther. 2011; 25:461-468.

37. Lyros O, Lamprecht AK, Nie L, Thieme R, Götzel K, Gasparri M, et al. Dickkopf-1 (DKK1) promotes tumor growth via Akt-phosphorylation and independently of Wnt-axis in Barrett's associated esophageal adenocarcinoma. Am J Cancer Res. 2019; 9:330-346.

38. Rozengurt E, Sinnett-Smith J, Eibl G. Yes-associated protein (YAP) in pancreatic cancer: At the epicenter of a targetable signaling network associated with patient survival. Signal Transduct Target Ther. 2018; $3: 11$.

39. González-Sancho JM, Aguilera O, García JM, Pendás-Franco N, Peña C, $\mathrm{Cal} \mathrm{S}$, et al. The wnt antagonist DICKKOPF-1 gene is a downstream target of beta-catenin/TCF and is downregulated in human colon cancer. Oncogene. 2005; 24:1098-1103.

40. Feng W, Xing D, Hua P, Zhang Y, Chen YF, Oparil S, et al. The transcription factor ETS-1 mediates proinflammatory responses and neointima formation in carotid artery endoluminal vascular injury. Hypertension. 2010; 55:1381-8.

41. Forghany Z, Robertson F, Lundby A, Olsen JV, Baker DA. Control of endothelial cell tube formation by notch ligand intracellular domain interactions with activator protein 1 (AP-1). The Journal of biological chemistry. 2018; 293:1229-1242.

42. Wang F, Marshall CB, Ikura M. Transcriptional/epigenetic regulator $\mathrm{CBP} / \mathrm{p} 300$ in tumorigenesis: Structural and functional versatility in target recognition. Cellular and molecular life sciences: CMLS. 2013; 70:3989-4008

43. Goodman RH, Smolik S. CBP/p300 in cell growth, transformation, and development. Genes \& Dev. 2000; 14: 1553-1577.

44. Bannister AJ, Oehler T, Wilhelm D, Angel P, Kouzarides T. Stimulation of c-Jun activity by CBP: C-jun residues Ser63/73 are required for CBP induced stimulation in vivo and CBP binding in vitro. Oncogene. 1995; 11:2509-2514

45. Bannister AJ, Kouzarides T. CBP-induced stimulation of c-Fos activity is abrogated by E1A. EMBO J. 1995; 14:4758-4762.

46. Yang C, Shapiro LH, Rivera M, Kumar A, Brindle PK. A role for CREB binding protein and p300 transcriptional coactivators in Ets-1 transactivation functions. Mol Cell Biol. 1998; 18:2218-2229.

47. Wasylyk B, Wasylyk C, Flores P, Begue A, Leprince D, et al. The c-ets proto-oncogenes encode transcription factors that cooperate with c-Fos and c-Jun for transcriptional activation. Nature.1990; 346:191-193.

48. Kim HY, Park JH, Won HY, Lee JY, Kong G. CBX7 inhibits breast tumorigenicity through DKK-1-mediated suppression of the Wnt/betacatenin pathway. FASEB J. 2015; 29:300-313.

49. Zhu N, Zhang D, Chen S, Liu X, Lin L, Huang X, et al. Endothelial enriched microRNAs regulate angiotensin II-induced endothelial inflammation and migration. Atherosclerosis. 2011; 215:286-293.

50. Gill JG, Langer EM, Lindsley RC, Cai M, Murphy TL, Murphy KM. Snail promotes the cell-autonomous generation of Flk1(+) endothelial cells through the repression of the microRNA-200 family. Stem cells and development. 2012; 21:167-176.

51. Wang C, Tan C, Wen Y, Zhang D, Li G, Chang L, et al. FOXP1-induced IncRNA CLRN1-AS1 acts as a tumor suppressor in pituitary prolactinoma by repressing the autophagy via inactivating Wnt/beta-catenin signaling pathway. Cell Death Dis. 2019; 10:499. 
52. Li S, Liu F, Pei Y, Wang W, Zheng K, Zhang X. Long noncoding RNA TTN-AS1 enhances the malignant characteristics of osteosarcoma by acting as a competing endogenous RNA on microRNA-376a thereby upregulating Dickkopf-1. Aging. 2019; 11:7678-7693.

53. Horie T, Baba O, Kuwabara Y, Chujo Y, Watanabe S, Kinoshita M, et al. MicroRNA-33 Deficiency Reduces the Progression of Atherosclerotic Plaque in ApoE-/- Mice. J Am Heart Assoc. 2012; 1:e003376.

54. Shan Y, Liu Y, Zhao L, Liu B, Li Y, Jia L. MicroRNA-33a and let-7e inhibit human colorectal cancer progression by targeting ST8SIA1. Int J Biochem Cell Biol. 2017; 90:48-58.

55. Talepoor AG, Kalani M, Dahaghani AS, Doroudchi M. Hydrogen Peroxide and Lipopolysaccharide Differentially affect the Expression of MicroRNAs 10a, 33a, 21, 221 in Endothelial Cells before and after Coculture with Monocytes. Int J Toxicol. 2017; 36:133-141. 